Meta

Journal des traducteurs

Translators' Journal

\title{
De Little Women de Louisa May Alcott aux Quatre filles du docteur March
}

\section{Les traductions françaises d'un roman de formation au féminin}

\section{Claire Le Brun}

Volume 48, numéro 1-2, mai 2003

Traduction pour les enfants

Translation for children

URI : https://id.erudit.org/iderudit/006957ar

DOI : https://doi.org/10.7202/006957ar

Aller au sommaire du numéro

Éditeur(s)

Les Presses de l'Université de Montréal

ISSN

0026-0452 (imprimé)

1492-1421 (numérique)

Découvrir la revue

Citer cet article

Le Brun, C. (2003). De Little Women de Louisa May Alcott aux Quatre filles $d u$ docteur March : les traductions françaises d'un roman de formation au

féminin. Meta, 48(1-2), 47-67. https://doi.org/10.7202/006957ar

\section{Résumé de l'article}

L'article examine sept traductions et adaptations françaises de Little Women de Louisa May Alcott (1868), actuellement accessibles aux jeunes lectrices en librairie ou en bibliothèque. Afin d'observer les représentations de la féminité qui y sont données à lire au lectorat francophone, l'analyse se centre sur le personnage de Jo, l'héroïne anti-conformiste qui n'hésite pas à exprimer ouvertement son refus des limitations imposées à la condition féminine. Il apparaît que la description physique et psychologique, les prises de parole et les actes du personnage ont subi, dans la plupart des versions françaises, des distorsions qui font de la Jo March française un personnage bien édulcoré.
Ce document est protégé par la loi sur le droit d'auteur. L’utilisation des services d’Érudit (y compris la reproduction) est assujettie à sa politique d'utilisation que vous pouvez consulter en ligne.

https://apropos.erudit.org/fr/usagers/politique-dutilisation/ 


\title{
De Little Women de Louisa May Alcott aux Quatre filles du docteur March: les traductions françaises d'un roman de formation au féminin
}

\author{
CLAIRE LE BRUN \\ Université Concordia, Montréal, Canada \\ lebrunc@vax2.concordia.ca
}

\begin{abstract}
RÉSUMÉ
L'article examine sept traductions et adaptations françaises de Little Women de Louisa May Alcott (1868), actuellement accessibles aux jeunes lectrices en librairie ou en bibliothèque. Afin d'observer les représentations de la féminité qui y sont données à lire au lectorat francophone, l'analyse se centre sur le personnage de Jo, l'héroïne anti-conformiste qui n'hésite pas à exprimer ouvertement son refus des limitations imposées à la condition féminine. II apparaît que la description physique et psychologique, les prises de parole et les actes du personnage ont subi, dans la plupart des versions françaises, des distorsions qui font de la Jo March française un personnage bien édulcoré.
\end{abstract}

\begin{abstract}
This article examines seven French translations and adaptations of Little Women by Louisa May Alcott (1868), nowadays available to young female readers in bookstores or in libraries. In order to study the representations of womanhood for the French-speaking readership, the analysis focuses on the character of Jo, the anti-conformist heroine who openly rejects the constraints imposed on women. It seems that most of the French versions depict a Jo March who has been significantly expurgated; her physical and psychological descriptions are distorted, as are her speech and actions.
\end{abstract}

\section{MOTS-CLÉS/KEYWORDS}

traduction et adaptation, classiques pour la jeunesse, littérature pour la jeunesse, roman de formation, représentations de la féminité

Peu de romans destinés à un jeune public féminin, tous pays et langues d'origine confondus, ont connu en France et dans les pays francophones un succès aussi unanime et aussi durable que Little Women (1868) de Louisa May Alcott. Depuis les premières traductions, qui suivirent de peu la publication $(1872,1880)$, le livre n'a cessé d'être diffusé sous divers titres et en diverses traductions et adaptations. Comme ce roman de formation suit l'évolution de quatre sœurs dont les âges s'échelonnent de seize à onze ans au début du récit, il nous a semblé pertinent de centrer cette étude sur les représentations de la féminité, particulièrement à travers le personnage de Jo, alter ego de l'auteure et héroïne implicite, qui entretient des relations conflictuelles avec les modèles de conduite autorisés.

Étant donné l'abondance des traductions françaises, il a fallu définir des critères de sélection. Il eût été intéressant d'adopter une approche diachronique et d'observer l'évolution des représentations de la féminité dans des démarches traductives 
s'échelonnant sur plus d'un siècle. Dans le cadre restreint de cet article, nous avons opté pour l'approche synchronique et le point de vue de la réception. Il nous a en effet paru justifié d'examiner la gamme de traductions françaises actuellement accessibles aux jeunes lectrices, soit en librairie, soit en bibliothèque. Après une enquête effectuée à partir de catalogues électroniques ${ }^{1}$, sept traductions ont été retenues pour une analyse contrastive: cinq actuellement disponibles dans les inventaires des libraires et deux autres plus anciennes, mais encore en circulation dans le réseau des bibliothèques.

Après avoir situé Little Women dans l'histoire de la littérature pour la jeunesse et de la littérature américaine, nous dresserons un panorama des traductions et adaptations successives du roman. Quant à l'analyse contrastive proprement dite des traductions retenues, nous l'avons ordonnée autour de ce que Philippe Hamon appelle les «systèmes normatifs-évaluatifs ", mis en place par le regard, la parole, les actes et gestes, les sensations et le sens esthétique, le savoir-vivre enfin des personnages (Hamon 1984: 103-217). Comme l'anticonformisme est le trait principal du personnage de Jo dans le texte source, une grille d'analyse prenant en charge la question des valeurs et des hiérarchies dans le texte littéraire s'imposait. L'hypothèse était que le personnage de garçon manqué, du tomboy, the man of the family, avec ses refus et ses ambitions, susciterait des résistances chez les traducteurs pour la jeunesse. L'analyse a bel et bien corroboré que c'était Jo qui inspirait les plus grands écarts dans les traductions, qu'il s'agisse du portrait physique et psychologique, ou des actions et des prises de parole les plus importantes.

\section{Little Women: «a girls' story»}

Née en 1832 en Pennsylvanie, Louisa est élevée dans une famille et un milieu peu ordinaires. Son père Bronson Alcott, philosophe largement autodidacte et pédagogue admirateur de Pestalozzi, côtoie Henry David Thoreau et Ralph Waldo Emerson. Durant l'enfance de l'auteure, il se lance dans diverses expériences communautaires. Utopie fouriériste, philosophie transcendentaliste, école expérimentale, l'enfant grandit dans ce climat intellectuel que l'on a pu appeler American Renaissance (Reynolds 1988). Ce cercle cosmopolite est en contact avec les grands mouvements socio-politiques européens. La mère, Abigail May, issue de l'establishment bostonien et d'une famille influente de l'Église unitarienne, admire la militante féministe Margaret Fuller (Woman in the Nineteenth Century, 1845). Selon la formule de Madeleine Stern, feminism was in Louisa May Alcott's genes (Stern 1996: vii). En outre, les Alcott sont des abolitionnistes convaincus. Avec l'aide de ses filles, Abigail May Alcott organise des ateliers d'alphabétisation pour les femmes noires (Barton 1996: 152). Pour Louisa May Alcott, que sa famille a placée au centre des grands débats de l'époque, le féminisme est la réforme des réformes. La critique féministe américaine l'a bien compris, qui considère Little Women comme l'un des premiers Female Bildungsromane américains (Langland 1983).

Dès sa parution en 1868, Little Women, or Meg, Jo, Beth, and Amy a été un bestseller. Écrit en deux mois, il est d'inspiration nettement autobiographique. L'entrée du journal de Louisa pour mai 1868 nous apprend que le roman est entrepris à la demande de son éditeur, qui lui demande une girls' story. Ainsi Louisa May Alcott, qui avoue ne pas beaucoup connaître et aimer les filles, à part ses sœurs, fait-elle partie du club select des écrivains pour la jeunesse mandatés par leurs éditeurs ${ }^{2}$ ! 
Little Women, qui assure instantanément la notoriété à la romancière, n'est pas sa première œuvre publiée. En 1854 était paru un recueil de contes, Flower Fables, écrit à l'origine pour la fille d'Emerson. Mais il faut surtout mentionner les récits abolitionnistes et féministes qui précèdent Little Women de quelques années, parmi lesquels Moods (1864) et Hospital Sketches (1863) qui transpose son expérience d'infirmière à Washington pendant la Guerre de Sécession. Devant le succès de Little Women, l'éditeur lui demande une suite: Good Wives paraît en 1869. Ce n'est que dans les années 1880 que les deux volumes seront réunis. Au fil des années, Louisa May Alcott poursuit l'histoire de la famille March: Little Men (1871), Jo's Boys (1886). Pour la jeunesse, elle signe aussi Eight cousins (1875), Rose in Bloom (1876) ${ }^{3}$ et Under the Lilacs (1877), et elle assume, entre ses deux voyages en Europe, la direction du journal Merry's Museum (1868-1869).

Bien que l'œuvre de Louisa May Alcott comporte plusieurs tendances: romans gothiques, fictions engagées sur le racisme et le féminisme, c'est comme auteure de Little Women qu'elle s'est acquis une renommée internationale: The Children's Friend donne lieu de nombreuses conférences et elle n'hésite pas à rencontrer son lectorat ${ }^{4}$. De son vivant, un million d'exemplaires du roman sont vendus. Little Women et sa suite Good Wives figurent dans toutes les collections de chefs-d'œuvre internationaux pour la jeunesse. Les auteurs de la récente Louisa May Alcott Encyclopedia soulignent le caractère résolument novateur du roman, particulièrement en ce qui a trait aux représentations féminines (Eiselein et Phillips 2001: 181) et, globalement, sa valeur littéraire ${ }^{5}$. En terminant, il peut être intéressant de rappeler que dans l'édition de 1948 des Children's Illustrated Classics, une note de l'éditeur fait pourtant allusion aux résistances que l'engagement nordiste de cette fiction pourrait susciter chez certains lecteurs. Laissant entendre que le livre n'est plus lu par les jeunes générations, ce paratexte encourage les lecteurs éventuels à aller au-delà de la leçon morale explicite ${ }^{6}$. À en juger par le nombre d'éditions et de traductions récentes des Quatre filles $d u$ docteur March, il est clair que ce dernier aspect - le traumatisme de la guerre civile étant peu probable - n'a pas diminué l'intérêt des lectrices francophones!

\section{Traductions et adaptations françaises}

Ce qui frappe dès qu'on aborde la réception de Little Women en milieu francophone, c'est le nombre et la constance des rééditions. Une consultation du catalogue de la Bibliothèque nationale de France donne une idée précise des traductions et adaptations «nouvelles », des "adaptations abrégées », des "abrégés » proposés par les divers éditeurs. Pour les années 1990, pas moins de neuf de ces versions plus ou moins nouvelles ont été déposées ${ }^{7}$. Puisque l'objectif n'est pas ici de faire l'histoire de la traduction française de Little Women, un survol des traductions successives suffira à éclairer notre propos.

La première traduction remonte à 1872 , soit quatre ans après la publication de l'original; publiée à Lausanne, elle est l'œuvre de Madame Rémy, connue également pour un manuel de français ${ }^{8}$. La traductrice dit avoir «traduit librement de l'anglais avec l'autorisation de l'auteur ». Puis, en 1880, Pierre-Jules Hetzel, célèbre éditeur français pour la jeunesse, publie sous le pseudonyme de P.-J. Stahl, Les Quatre filles du docteur Marsch [sic] d'après L.M. Alcott, pour son «Magasin d'éducation et de récréation ", fondé en 1864. P.-J. Stahl a joué un rôle décisif dans la transmission de 
Little Women en domaine francophone. Cette adaptation, plus ou moins retouchée par les éditeurs et des adaptateurs anonymes, continue d'avoir cours et nous l'avons incluse dans le choix de traductions. Elle a imposé au récit des distorsions qui n'ont pas été rectifiées depuis.

Ainsi c'est Stahl qui impose cette fiction du «docteur» March, en faisant jouer au père dans l'armée nordiste, non pas le rôle d'un aumônier, mais celui d'un méde$\operatorname{cin}^{9}$. Version plus acceptable dans une France républicaine où s'affrontent les tenants du catholicisme ultramontain et de la laïcité, peu réceptive dans son ensemble au mariage des guides spirituels. L'éditeur estimait en effet que le livre «tel qu'il était, n'aurait pu $[\ldots]$ réussir en France ${ }^{10} »$. Si les traductions récentes ont corrigé l'erreur dans le texte, le titre demeure inchangé, et les lectrices francophones continuent de croire que le père des sœurs March est docteur en médecine. Son absence dans le premier tome permet d'ailleurs de ne pas se poser trop de questions. La seconde modification substantielle apportée au texte est le gommage systématique de l'intertexte bunyanien. Chez Stahl, la Morale familière remplace le Pilgrim's Progress. Or, ce texte clé de la spiritualité protestante (1678), évoqué dès le premier chapitre, imprime son mouvement au texte d'Alcott; plusieurs titres de chapitres, complètement modifiés dans la traduction, y renvoient explicitement: «Playing Pilgrims» (ch. 1), «Burdens» (ch. 4), «Jo meets Apollyon» (ch. 8), etc. Enfin l'adaptateur, qui de son propre aveu ne connaissait pas le second tome, a imaginé un dénouement, dont s'inspirent quelques adaptations actuelles. La maison Hachette, qui racheta le fonds Hetzel, a publié l'adaptation de Stahl, sensiblement remaniée, dans les collections «Idéal-Bibliothèque» et "Bibliothèque Verte» ${ }^{11}$. L'éditeur belge Casterman propose une réécriture anonyme basée sur cette adaptation, qui est dans l'ensemble plus fidèle au texte de 1880 que ladite traduction de Stahl publiée par Hachette, comme nous le verrons plus loin. On voit donc que les textes issus de l'adaptation de la fin du XIX ${ }^{\mathrm{e}}$ siècle connaissent encore une importante diffusion.

Dans les années 1930 et 1940, Little Women a été traduit et édité pour un public adulte $^{12}$. Au cours des années 1980, apparaissent, dans des collections destinées à la jeunesse, des textes plus conformes à l'éthique moderne de la traduction, qui ne se permettent pas de coupures ou d'ajouts. Nous en incluons deux dans le corpus, celle de Paulette Vielhomme-Callais pour Gallimard (Folio-Junior et Gallimard Jeunesse) et celle de Maud Godoc pour Castor-Poche/Flammarion.

Quelques mots suffiront à présenter les six adaptations et traductions sélectionnées pour l'analyse comparative, que nous classons par ordre chronologique: l'adaptation de P.-J. Stahl rééditée, avec diverses modifications, par Hachette dans «Idéal-Bibliothèque»; la traduction anonyme ${ }^{13}$ des éditions G.P., collection "Rouge et Or»- ces deux ouvrages sont épuisés mais connaissent encore une grande diffusion dans les bibliothèques scolaires et municipales qui n'ont pas toujours fait l'acquisition de nouvelles éditions -; l'adaptation anonyme de Casterman, basée sur celle de P.-J. Stahl; la traduction de Paulette Vielhomme-Callais pour Gallimard; la traduction de Maud Godoc pour Castor-Poche; enfin il nous a paru intéressant d'inclure une adaptation récente, celle de Rémi Simon pour Nathan. Nous avons également examiné celle de Chantal Baligand pour les éditions Hemma, mais nous n'avons pu l'utiliser dans une comparaison systématique, étant donné l'importance des coupures apportées au texte (14 chapitres sur 23). Dans les passages traduits, on 
peut observer une dépendance à l'égard du texte de P.-J. Stahl. Nous nous y référons ponctuellement.

Avant d'examiner les traductions sous l'angle particulier que nous avons choisi, quelques commentaires plus généraux s'imposent. La traduction parue chez G.P. s'avère dans l'ensemble la plus littérale; elle n'a pas craint de restituer les références à Bunyan en les explicitant ${ }^{14}$. Le texte de Casterman, basé sur P.-J. Stahl, comme le prouvent de nombreuses formulations identiques, notamment dans les titres de chapitres, comporte des omissions, mais conserve aussi de son modèle certains ajouts de l'adaptateur qui ont été supprimés chez Hachette. Ainsi, au chapitre 3, quand Meg et Jo se rendent au bal, il précise que Madame March n'a pu les accompagner, car elle était souffrante et qu'elle a demandé à une amie de veiller sur ses filles ${ }^{15}$. Quand Jo rend visite à Laurie dans sa chambre, elle glose que le jeune voisin a été malade et qu'il n'est qu'un "petit garçon $»^{16}$. À la fin du chapitre 12, se trouve une longue addition sur les vertus de l'Amérique et l'indépendance des jeunes filles américaines ${ }^{17}$. Par ailleurs, cette traduction a une nette tendance à la miniaturisation, désignant régulièrement les protagonistes comme des «petites» filles et des "petits " garçons ${ }^{18}$. Dans l'ensemble, elle ennoblit le caractère de Jo, comme nous le verrons plus loin. L'adaptation de Rémy Simon pour Nathan se permet des ajouts sur le ton et les gestes dans les phrases introductives du discours rapporté, modifiant imperceptiblement le caractère des personnages. Ainsi, dans le chapitre 1, Meg regarde "d'un œil critique » sa vieille robe "encore très mettable », Jo siffle « d'un air méprisant». Amy est qualifiée d' «insupportable». Quant à la servante, elle est régulièrement appelée la «vieille» Hannah. Enfin les traductions de Paulette Vielhomme-Callais pour Gallimard et de Maud Godoc pour Castor-Poche se sont attachées à rendre intégralement et fidèlement l'œuvre d'Alcott. Nous examinerons leurs choix de traduction sur la question de la féminité.

\section{Une jeune féministe américaine en français}

Comme il a été dit plus haut, la «poétique du normatif» proposée par Philippe Hamon a servi de cadre théorique à notre analyse des textes source et cible. Nous avons concentré l'analyse des traductions sur les passages où le personnage de Jo se définissait ou était vue par les autres, sur ceux où elle se distinguait par les actions ou l'expression des goûts et de la volonté: les «nexus normatifs» (Hamon 1984: 220). Les travaux en linguistique de l'énonciation sur les marques de subjectivité nous ont aussi guidés. Nous avons particulièrement examiné la traduction - et souvent la nontraduction - des phrases introductives du discours rapporté, porteuses d'indication sur les sentiments, la gestuelle, la voix des personnages. Dans les portraits et les descriptions, une attention particulière a été accordée aux termes évaluatifs, particulièrement les adjectifs (Kerbrat-Orecchioni 1980: 83-100), dont la traduction donne lieu à des choix très révélateurs. Pour la brièveté, nous référerons désormais aux traductions et traductions par les abréviations: H (Hachette), GP (G.P.), C (Casterman), N (Nathan), FJ (Folio Junior) et CP (Castor Poche), initiales de leurs éditeurs respectifs, et à l'original par l'abréviation LW, toutes suivies du numéro de page. L'abréviation S est occasionnellement utilisée pour envoyer à l'adaptation de Stahl de 1880. Des tableaux présentés en annexe permettent de rendre l'analyse contrastive plus concise. 


\section{a) Portrait physique et psychologique}

Le premier chapitre comporte un portrait des quatre sœurs; il est frappant de constater que c'est le portrait de Jo qui a été le plus diversement traduit. Les divergences apparaissent notamment dans la traduction des nombreux adjectifs qui permettent aux lectrices de se représenter l'héroïne (tableau 1). Les traducteurs français tentent souvent d'atténuer ce qui dans l'apparence physique de l'héroïne peut leur sembler outré ou disgracieux, en se conformant à leurs propres canons de la beauté féminine. Ainsi le comical nose devient «retroussé» ou "petit», la bouche (decided) est «bien dessinée». Ces retouches, particulièrement évidentes dans les versions dérivées de l'adaptation de 1880, se retrouvent encore dans les plus récentes. Ainsi, dans CP (1995), Jo est «très élancée ». L'hésitation entre «mince» et «maigre» est également révélatrice. Les ajouts de l'adaptation de N (1988) soulignent la différence de Jo en la comparant à son aînée Meg, explicitant ainsi ce qui n'est qu'esquissé dans le texte source ${ }^{19}$ : la seconde est «nerveuse » et ses mains sont «quelque peu masculines». Il est à noter que les portraits sont omis dans l'adaptation de C.

À propos de l'adjectif tall, le premier évaluatif appliqué à Jo, il convient de remarquer que le même adjectif est utilisé par Alcott pour décrire Madame March lors de sa première apparition (a tall, motherly lady, LW: 9). Dans la suite du texte (chapitre 8), on apprendra que cette ressemblance physique se double d'une ressemblance morale, car Jo a hérité du tempérament impétueux de sa mère. Or, cet indice important a été gommé dans quatre des six traductions analysées.

Généralement décrits dans les phrases introductives du discours rapporté, le ton de voix, les expressions et la gestuelle de Jo ont été dans une proportion importante effacés des traductions. Alcott fait un grand usage de ces segments textuels, que Gérald Prince appelle de façon éclairante le «discours attributif» (Prince 1978), caractérisant la voix, l'état d'esprit du personnage, sa relation aux autres. La répétition de certains adverbes ou syntagmes prépositionnels en association avec la prise de parole de tel ou tel personnage a pour effet de fixer ses traits de caractère. Ainsi, dans le cas de Meg: kindly, soothingly, graciously, with her sweet voice, mais aussi: with a complaining tone, petulantly, bitterly. Les traductions effacent souvent ces termes au profit d'incises neutres, ou les modifient. Ces amputations et distorsions s'appliquent à l'ensemble des protagonistes, et on ne peut dire que le personnage de Jo ait subi un traitement particulier à cet égard. Il n'en demeure pas moins que les modalisateurs de la parole de Jo - briskly, impetuously, sharply, impatiently, fiercely - n'apparaissent que sporadiquement dans les textes français.

Au chapitre 2, dans la scène du matin de Noël où les quatre sœurs découvrent un exemplaire du Pilgrim's Progress, l'instance narratoriale décrit Jo lisant, with the quiet expression so seldom seen on her restless face (LW : 16). Seule la traduction de FJ (27) a traduit restless (visage mobile). La gestuelle du «garçon de la famille» ou du «fils Jo» est également l'un des points névralgiques de la traduction française. Exemple caractéristique, dans le chapitre premier où Amy reproche à sa sœur d'utiliser des termes argotiques, la phrase introductive du discours rapporté se lit: observed Amy, with a reproving look at the long figure stretched on the rug (LW: 3 ). Dans les quatre traductions qui ont conservé le dernier segment, on hésite entre l'élégant: une «longue silhouette étendue sur le tapis» (C: 9), la pointe de reproche: «longue silhouette étendue de tout son long...» (CP: 7), «grand corps étendu...» (GP:11) et la nette 
réprobation, endossant ainsi le point de vue d'Amy: «à son aînée, toujours vautrée sur le tapis» (FJ: 12) Un peu plus haut, l'une des paroles de Jo est suivie de l'indication: cried Jo, examining the heels of her shoes in a gentlemanly manner (LW:2). Seules les deux traductions récentes l'ont prise en compte et rendue par "mâle assurance» $(\mathrm{FJ}: 10)$ et «aplomb tout masculin» $(\mathrm{CP}: 6)$. Les exemples pourraient être multipliés. En voici un dernier des plus probants: au chapitre 3, l'héroïne rajuste sa toilette avant de faire son entrée à son premier bal. La phrase introductive d'Alcott se lit: returned Jo, giving her collar a twitch, and her head a hasty brush (LW: 34). Les traductions atténuent généralement la brusquerie des gestes de Jo, que l'on doit imaginer, «mettant sa collerette droite et donnant un dernier regard à sa coiffure » $(\mathrm{H}: 23-24$ et $\mathrm{C}: 26)$, «donnant un coup de pouce à son col et un coup de brosse à ses cheveux» (GP: 36). Seules les deux traductions récentes restituent cet aspect de la présence physique de l'héroïne: «tirant sur son col d'un coup sec et rectifiant sa coiffure à la va-vite» (CP: 39), «qui passa vaguement la main sur son chignon et rajusta son col d'une pichenette» $(\mathrm{FJ}: 48)$.

Le chapitre 20 comporte des notations décrivant précisément la gestuelle de Jo, qui ont été soit gommées soit atténuées dans la traduction, comme le montrent les deux exemples qui suivent. L'adolescente a une conversation orageuse avec sa mère à propos des amours naissantes de son aînée. Dans le feu de la conversation, elle joue avec ses cheveux, objet sémiotique particulièrement important dans le texte; en effet, le chapitre 1 les décrit comme sa seule beauté (her one beauty, LW: 5) et elle vient de les sacrifier pour apporter une aide financière à son père malade (chapitre 15). L'indignation de Jo s'exprime dans la façon dont elle tire sur ses cheveux: And Jo pulled her hair again with a wrathful tweak (LW:261). La colère, trait constitutif du caractère de Jo, comme nous le verrons plus loin, a été atténuée dans certaines traductions (tableau 2). Une seulement (GP) traduit: "avec fureur», deux préfèrent le désespoir ( $\mathrm{H}$ et $\mathrm{C}$ ), deux l'adverbe "rageusement», dont le contenu sémantique est nettement affaibli dans les emplois modernes et souvent associé aux manifestations enfantines. Le geste même de tirer est corrigé dans certaines traductions: «se passer la main dans les cheveux» $(\mathrm{H}$ et $\mathrm{C})$, «fourrager» $(\mathrm{GP})$ et le diminutif «tortiller» $(\mathrm{CP})$. Chez $\mathrm{H}$ et $\mathrm{C}$, la gestuelle est transposée à l'expression du visage. Plus loin, le mouvement de Jo se levant est comparé à celui d'un pantin (said Jo, unfolding herself, like an animated puzzle, LW : 264); l'image perçue comme peu féminine est gommée dans les versions françaises à l'exception des deux traductions récentes.

Le registre linguistique de Jo est stigmatisé dès le premier chapitre. Au fil du texte, sa mère et ses sœurs citent ses écarts de langage, entre guillemets. Bien que la liberté verbale soit, avec le tempérament impétueux, l'un des traits constitutifs de l'étiquette du personnage ${ }^{20}$, les traductions ont souvent renoncé à traduire son juron préféré, Christopher Colombus ${ }^{21}$ ! (chapitres 12 et 17). C le tempère d'un «Priez pour nous!»(75). Curieusement, les traductions récentes proposent des équivalents: «Nom d'un petit bonhomme» (FJ: 203), "Sacré nom d'un pipe» (CP: 172), «Bon sang de bonsoir» $(\mathrm{N}: 94)$, traductions d'un ciblisme très hexagonal sur la justesse desquelles on pourrait s'interroger.

Pour le portrait psychologique de Jo, nous nous attarderons sur les traductions du chapitre 8: Jo meets Apollyon, où l'adolescente fait l'expérience des conséquences désastreuses de sa colère. Il faut rappeler que Little Women consacre un chapitre particulier à chacune des sœurs March et que celui qui est consacré à Jo est particulièrement 
dramatique. Or, ce chapitre donne lieu à des écarts notables dans les traductions. Rappelons brièvement les événements. Amy, furieuse que ses aînées ne l'aient pas emmenée au théâtre et particulièrement ulcérée par les rebuffades de Jo, brûle le cahier où celle-ci avait soigneusement recopié des contes de fée qu'elle espérait voir publiés un jour. Le lendemain, Jo, toujours en colère, omet de mettre Amy en garde contre les dangers de la glace trop mince et sa jeune sœur manque de se noyer. Le chapitre est d'autant plus central que la perte du cahier touche aux ambitions les plus profondes de l'héroïne. Il peut se diviser en trois séquences : la colère de Jo en découvrant la calamité; l'accident d'Amy; la conversation au cours de laquelle Madame March avoue à sa seconde fille ses propres faiblesses. Le titre du chapitre, référence au Pilgrim's Progress, a été sensiblement modifié dans trois des traductions: "Double choc» $(\mathrm{H}$ et $\mathrm{C})$, «Où Jo se met en colère" $(\mathrm{N})$ ou explicité «Jo rencontre le démon Apollyon» (GP et FJ). Le traducteur de G.P. fait œuvre didactique en glosant her bosom enemy (LW : 96) : "son ennemi intérieur, son Apollyon, comme le vieux Bunyan l'appelle dans le voyage du Pèlerin » (GP: 75).

Le caractère, les réactions, les motivations de Jo apparaissent sensiblement différentes selon les traductions. La troisième partie varie beaucoup en importance. Complètement effacée chez $\mathrm{C}$ et $\mathrm{N}$, elle est condensée très allusivement dans la formule: "tout le monde a ses faiblesses» chez GP. H résume, mais conserve l'essentiel des confidences de la mère en deux paragraphes. Seuls $\mathrm{F}$ et $\mathrm{C}$ donnent le texte au complet, y compris l'aveu de madame March au sujet des sacrifices qu'elle a dû consentir pour sa patrie. L'existence d'un modèle maternel fort, voire héroïque - personnage dont la charge positive se communique à l'héroïne qui lui ressemble - est donc gommée dans la plupart des textes français actuellement offerts aux jeunes lectrices.

Dans la première partie, la violente réaction de Jo à l'égard d'Amy est diversement traduite, et souvent tempérée, comme le montrent les extraits comparés (tableau 3). Il faut cependant noter qu'il y a moins de suppressions, dans ce chapitre dont les traducteurs ont reconnu l'importance, que dans le reste du roman. Le tableau permet par ailleurs d'observer la parenté de $\mathrm{H}$ et de $\mathrm{C}$ dérivés de Stahl. La traduction de $\mathrm{H}$ est la plus édulcorée: Amy, secouée, perd la respiration et Jo s'en va " cacher» son chagrin. Le geste final, le parting box, semble poser problème à certains traducteurs. Supprimé chez Hachette, il réapparaît sous forme de «tape» dans le texte de Casterman. Rémi Simon (N) a arrêté le bras de Jo. Ailleurs, on parle de gifle, maîtresse même (GP) ou de taloche. Curieusement, aucun des traducteurs et traductrices n'a jugé utile de faire mention de l'oreille d'Amy. On notera aussi qu'une seule traduction (FJ) mentionne la nature violente (hot temper) de l'héroïne.

Des traductions minimisent également la profondeur du désespoir de Jo, quand elle voit son œuvre littéraire détruite. Les ambitions personnelles du personnage sont nettement restreintes chez Hachette où il s'agit de venir en aide à sa mère: "rêve innocent». Casterman renchérit avec la mention d'un journal pour bébés! Plus loin dans le chapitre, quelques ajouts des traductions tendent à ennoblir le personnage de Jo. Dans l'adaptation de Stahl, Meg dit à Amy que Jo « est peut-être la meilleure de nous toutes» $(\mathrm{H}: 62 ; \mathrm{C}: 57)$. Rémi Simon ajoute qu'elle n'est pas rancunière $(\mathrm{N}: 79)$.

La scène centrale de l'accident sur la rivière gelée et le rôle de Jo donnent également lieu à des distorsions (tableau 4). Seules les deux traductions récentes osent plonger dans les mauvais sentiments de l'héroïne. Les paroles du petit démon sont diversement interprétées. La traduction de Casterman déculpabilise l'héroïne. Rémi 
Simon (N) évacue l'idée du démon, et traduit dans un registre familier sa voix qu'il prête à l'héroïne. Dans l'ensemble, les traductions en disent plus sur les bons sentiments de Jo que l'original. Strange feeling est traduit par «inquiétude» par G.P. et Nathan. Casterman précise: «inquiète sans vouloir le paraître» et ajoute "son bon cœur l'emportant», "se retourna vivement pour avertir Amy». Ces extraits suffiront à montrer que la traduction résiste dans l'ensemble à exprimer la violence des sentiments de Jo.

\section{b) Jo vue par son entourage}

Viennent compléter le portrait de l'adolescente anti-conformiste les appréciations de son entourage: ses parents, ses sœurs, l'acariâtre tante March, les voisins amis M. Laurence et son petit-fils Laurie; ces jugements sont véhiculés par la voix narratoriale ou par les personnages eux-mêmes. Au chapitre 2, les sœurs qui vont porter leur déjeuner de Noël à une famille dans la misère sont comparées à des anges par l'heureuse bénéficiaire. La voix narratoriale commente que le compliment fait particulièrement plaisir à Jo qui s'est fait traiter de diable depuis sa naissance. Voilà un point où les traductions diffèrent. Deux des traductions $(\mathrm{GP}, \mathrm{N})$ ont supprimé cette remarque, deux autres $(\mathrm{H}: 18, \mathrm{C}: 19)$ l'adoucissent : "mais surtout Jo qui avait mérité maintes fois (souvent reçu C) dans son enfance le sobriquet de "petit diable" ». Jo a su s'attirer l'affection de tante March qui l'a engagée comme dame de compagnie: Something in her comical face and blunt manners struck the old lady's fancy (LW: 47). L'adjectif se révèle encore une fois l'un des points névralgiques de la traduction du personnage de Jo: le syntagme comical face dérange autant que le comical nose du chapitre 1. Même dans les traductions récentes, il est adouci par les hypocoristiques «frimousse» $(\mathrm{FJ})$ ou «minois» $(\mathrm{CP})$. P.-J. Stahl $(\mathrm{H})$ avait résumé les deux caractéristiques par «originalité». Rémi Simon $(\mathrm{N})$ a rendu comical face par «visage franc». Le chapitre est supprimé chez Casterman. La traduction de G.P. est comme dans bien des cas la plus littérale (tableau 5).

Les manières originales de Jo plaisent aussi à leur voisin Laurie dès la première rencontre, manières que les traductions ont hésité à qualifier: Jo's gentlemanly demeanour (LW: 37) est omis par H et N, C (29) traduit «son air gentleman", les autres optent pour les manières garçonnières (GP:38, FJ : 53) ou l'attitude garçonne (CP : 43). Enfin l'un des jugements les plus significatifs portés sur Jo est celui d'une jeune Anglaise visitant l'Amérique (chapitre 12). Le passage vaut d'être examiné en détail (tableau 6). Il est curieux qu'aucune des traductions n'hésite à qualifier Jo de bizarre ou d'étrange par le truchement de la Britannique Miss Kate, mais que certaines hésitent à lui accorder de l'intelligence, la préférant «sympathique» (G.P.) ou instruite $(C)$. Les exemples pourraient être multipliés, mais nous espérons avoir montré que la caractère audacieux et extrême du personnage a été estompé, et que l'énergie extraordinaire de la queer girl ne passe pas toujours en traduction.

\section{c) Goûts, rêves et ambitions}

L'expression de la volonté de Jo est un aspect que les traductions se révèlent récalcitrantes à transmettre. Jo entend dire ce qu'elle pense (good, strong words, LW : 45), déteste attendre, veut agir sur les choses et se soucie peu du qu'en-dira-t-on. Elle 
exprime son désir de liberté en toute occasion. Bien que cela ait été respecté, globalement, dans les traductions françaises, certains rêves de Jo ont semblé choquer. Au chapitre 14, après une bonne course avec son ami Laurie, qui lui vaut la réprobation de Meg, l'adolescente s'exclame: "I wish I was a horse; then I could run for miles in this splendid air, and not lose my breath. It was capital, but see what a guy it's made $m e . . . »(\mathrm{LW}: 198)$. La tenue vestimentaire de Jo a en effet souffert de l'exercice, et elle a perdu toutes ses épingles à cheveux. L'allusion au cheval a été gommée par GP et N. Chez $\mathrm{H}(105)$ et $\mathrm{C}(88)$, à la suite de Stahl, on choisit un animal plus associé à la féminité: "Je voudrais être une gazelle, ou un cheval...», "Ou même un cheval», concède $C$, plus fidèle au modèle ( $S$ : XXXII, 61).

Les ambitions littéraires de Jo, composante essentielle du système de valeurs mis en place par le roman, sont souvent minimisées dans les traductions. Cette censure, dont nous avons déjà touché un mot au sujet du chapitre 8 , s'observe encore au chapitre 14. Quand Jo avoue à Laurie qu'elle a soumis deux textes à un journal, le texte de $\mathrm{H}$ (102) et de C (85) suivant Stahl, se lit: «j'ai donné deux histoires de ma façon au directeur du Journal des Enfants». La fin du chapitre a subi d'importantes distorsions. Quatre des traductions résument considérablement la fin de l'épisode. La réaction de fierté de Madame March devant le succès de sa fille est escamotée chez $\mathrm{H}$ et $\mathrm{N}$ qui arrêtent le chapitre aux exclamations de joie des sœurs. Le passage est supprimé chez GP également. Chez C (91), Madame March «ne se montra pas mécontente», car «l'histoire était gentille et convenable; elle faisait honneur aux sentiments moraux de l'auteur» (S: XXXII, 61). Les dernières lignes du chapitre: "For to be independent, and earn the praise of those she loved, were the dearest wishes of her heart [...]» (LW: 203), si importantes pour la compréhension globale du texte, n'apparaissent pas chez H, N, C. Chez GP, la fierté devient de l'approbation. Encore une fois, seules les deux traductions récentes traduisent «faire honneur» (FJ : 254) et «faire la fierté» (CP: 215). Il est curieux aussi que la fin de la phrase précédente où Jo explique qu'elle aimerait aider ses sœurs (support myself and help the girls, LW: 203) est traduite par une généralisation - «aider les autres» (GP:131), «aider les miens» (CP: 215) - dans deux des traductions qui l'ont conservée. On peut faire un rapprochement avec les propos d'Amy au chapitre 15: la plus jeune sœur, qui rêve d'une carrière artistique, assure que dans dix ans Jo et elle auront fait la fortune de la famille. Cette phrase a été supprimée dans quatre des traductions (H, GP, C, N). Il semble que, tout autant que la personnalité affirmée de Jo, ses ambitions professionnelles heurtent en traduction!

Le rêve de liberté et d'indépendance s'exprime aussi quand Laurie, fâché contre son grand-père, propose à Jo de partir avec lui pour Washington (chapitre 21). Dans le texte d'Alcott, l'héroïne est fortement tentée par l'aventure et se bouche même les oreilles pour ne pas entendre la suite des projets séduisants. GP occulte complètement ce moment de rêverie de Jo, qui propose tout de suite à Laurie de l'aider à se réconcilier avec son grand-père. $\mathrm{H}$ (162) donne l'essentiel du passage et explicite même she was tired of care and confinement (LW : 273) par «elle était fatiguée de sa réclusion auprès de Beth ». Reprenant exactement son modèle (S: XXXII, 283), C (163) rend compte de la tentation de Jo, mais moralise par des ajouts: «l'étourdie», «ses yeux tombèrent heureusement [sur sa maison]», «ce fou de Laurie», etc. N (167) résume en quatre lignes, concluant avec autorité: «son bon sens naturel reprit le dessus». Encore une fois, les deux traductions récentes sont plus fidèles. 
Les lamentations de Jo sur les restrictions imposées à la condition féminine (chapitres $1,3,5,10,16,20,21$ ) ont été traduites dans l'ensemble du texte sans trop d'amplification ni d'atténuation. Toutefois, dans cette scène du chapitre 21 , les différences dans les traductions françaises signalent un point névralgique. L'emphase de $\mathrm{H}$ et surtout de $\mathrm{C}$ contraste avec le détachement de $\mathrm{N}$, alors que GP, comme on l'a vu, n'a pas jugé bon de s'appesantir sur la tentation de Jo (tableau 7).

\section{d) Le point d'aboutissement}

Que devient l'héroïne contestataire à la fin du roman? Plusieurs réponses sont données selon les points de vue narratifs. Jo elle-même s'avoue très mécontente et ne fait que se résigner aux fiancailles de Meg qui réjouissent toute la famille. Elle sent qu'elle n'a aucune prise sur les cadres sociaux. Le parcours de l'adolescente au cours de l'année délimitée par les deux fêtes de Noël est décrit par monsieur March à son retour. Il dit ne plus reconnaître le «fils Jo» qu'il a laissé l'année précédente. Les transformations qu'il note concernent la tenue vestimentaire, le langage, les gestes, la voix, le développement du sentiment maternel. La traduction de la fin de son discours présente quelques écarts (tableau 8).

Our black sheep, la métaphore paternelle est on ne peut plus claire. L'image a été supprimée dans trois traductions $(\mathrm{H}, \mathrm{C}, \mathrm{N})$. "Tête ronde» de $\mathrm{H}$ et $\mathrm{C}$ renvoie allusivement au sacrifice des cheveux au chapitre 15 . S’il énonce quelque regret, Monsieur March se félicite de voir sa fille entrer dans le rang. Les traductions françaises n'ont ni forcé la note, ni atténué la portée du discours. L’adaptation de Rémi Simon (N: 177) ajoute cependant, au nombre des progrès de Jo: «et dont les récits sont publiés dans les journaux!». Observons aussi la façon dont les traductions ont rendu les trois adjectifs strong, helpful, tender-hearted (woman) qu'il oppose à wild (girl). Stahl (repris par C) avait opté pour quatre adjectifs «tendre, dévouée, forte encore, mais civilisée ». H omet l'idée de force, tandis que l'adaptation de Simon la conserve. Dans les deux traductions récentes, proches l'une de l'autre, on a préféré le courage. Il faut noter par ailleurs que la traduction «brebis galeuse» de FJ et CP est nettement plus péjorative que "mouton noir».

L'adaptation de P.-J. Stahl, encore très diffusée par l'intermédiaire d'Hachette (Idéal-Bibliothèque et Bibliothèque verte) et de Casterman, prend beaucoup de libertés avec la fin de l'histoire ${ }^{22}$. H ajoute un chapitre «Perspectives d'avenir» qui a fonction d'épilogue. La lectrice y apprend que Jo « renonçant à écrire toutes les histoires qui lui passaient par la tête, s'adonna avec ardeur à la confection du trousseau de sa sœur» (H: 184)! C (185-187) ajoute au dernier chapitre des détails touchants, comme ce geste de Beth montant sur les genoux du fiancé de Meg, Monsieur Brooke, pour lui demander à l'oreille s'il rendra sa sœur heureuse. Monsieur March annonce que Meg se mariera dans deux ans, ce qui lui permettra de perfectionner son éducation : «Cela vous donnera aussi, Jo, l'occasion de compléter la vôtre». En quel sens? peut-on se demander. Et l'adaptateur conclut : "Monsieur March avait appris à Jo tout ce qu'il y avait à apprendre». Enfin, la résistance de Jo au mariage de sa sœur a complètement disparu grâce à ses «sages réflexions ", comme elle l'explique à Laurie. Mais P.-J. Stahl allait plus loin encore en mariant Jo à Laurie. Fort curieusement, l'adaptation toute récente de Chantal Baligand réintroduit cette fin improbable et en totale contradiction avec le second tome. Elle reprend en effet, en l'abrégeant, l'épilogue «Quatre ans 
après » de 1880, où le jeune dandy Laurie devenait fermier pour l'amour de Jo, avec la bénédiction de son grand-père (Baligand 2002: 153-156). Ainsi, le dénouement matrimonial - par une union que Louisa May Alcott s'était toujours refusé à faire, en dépit des prières de ses lectrices ${ }^{23}$ - est-il réactivé à l'aube du $\mathrm{XxI}^{\mathrm{e}}$ siècle!

\section{La seconde fille du «docteur» March, pâle reflet de l'héroïne d'Alcott?}

Il serait à peine exagéré de dire que des textes bien différents circulent actuellement sous le titre des Quatre filles du docteur March. Sur les six adaptations et traductions que nous avons analysées en détail, quatre présentent des distorsions marquées par rapport à l'original. L'amputation de chapitres (de trois à cinq), la suppression de passages complets ou d'éléments significatifs des phrases y a pour pendant des gloses interprétatives, particulièrement dans l'adaptation anonyme de Casterman, la plus fidèle à Stahl. Nous avons observé en particulier de quelle façon le « discours attributif» des paroles des personnages était souvent privé des notations sur le ton, l'expression ou le geste. En outre, nous avons eu la surprise de découvrir que, parmi les versions les plus récemment éditées, se trouvaient aussi bien des traductions soucieuses de transmettre le sens global de l'œuvre que des adaptations qui la dénaturaient.

Cette étude des représentations de la féminité en traduction française, que nous avons centrée sur le personnage de Jo, l'adolescente éprise d'action et d'indépendance, fait apparaître - même dans les traductions récentes, sous certains aspects une édulcoration de l'héroïne. Les traits physiques, la parole, les actions et les ambitions ont subi d'importantes retouches dans les versions françaises, qu'il s'agisse des adaptations, mais aussi des traductions. Les sœurs March continuent de fasciner les lectrices francophones, car il reste toujours quelque chose des grandes œuvres, même dans les adaptations les plus désinvoltes! Mais on peut déplorer que le premier contact avec ces héroïnes américaines du XIX ${ }^{\mathrm{e}}$ siècle soit bien souvent faussé et appauvri, selon l'édition qui leur sera accessible en bibliothèque ou en librairie.

\section{NOTES}

1. L'enquête, effectuée à partir des ressources disponibles à Montréal, nous paraît toutefois représentative de la diffusion actuelle des traductions françaises de Little Women. Il est à noter qu'aucune traduction n'a été publiée à ce jour en Amérique francophone; une telle entreprise présenterait de l'intérêt en raison des liens étroits entre la culture source et la culture cible.

2. «Mr. N. [Niles] wants a girls'story, and I begin “Little Women”. Marmee, Anna, and May all approve my plan. So I plod away, though I don't enjoy this sort of thing. Never liked girls or knew many, except my sisters, but our queer plays and experiences may prove interesting, though I doubt it. » (Myerson \& Shealy 1989: 165-166) Sur les «vocations» d'écrivains pour la jeunesse suscitées par les éditeurs, voir par exemple Shavit 1986: 33-57; Grenier 1993.

3. Pour juillet-août 1876, elle note dans son journal: "Get an idea and start "Rose in Bloom", though I hate sequels.» (Myerson \& Shealy 1989: 201)

4. L'entrée de son journal pour les mois de juin à août 1875 fait état de 92 visiteurs: «Fame is an expensive luxury.» (Myerson \& Shealy 1989: 196)

5. «Particularly when studied within its critical and cultural contexts, Little Women emerges as a substantial, multidimensional literary text.» (Eiselein et Phillips 2001: 182)

6. «Indeed, one can guess that with the passage of time, when a new domestic culture is sufficiently established not to be embarrassed by reminders of the past, youngsters no less than adults will return to it with fresh enthusiasm as a charming and authentic record of New England life in the Civil War period.. [...] For the contemporay reader, what is perhaps most striking about Little 
Women, even more archaic that its social scene, is its overt moral emphasis. " (Alcott 1948: couverture arrière)

7. Pour la dernière décennie, outre les traductions sélectionnées pour cette étude, il faut mentionner: une «traduction nouvelle» d'Anne Joba avec dossier de Nicole Bon aux éditions France loisirs, Paris, 1992; une «adaptation abrégée» anonyme aux Éditions du Tournesol, Limay, 1994; une traduction anonyme avec dossier de Françoise Gomez chez Pocket Junior, Paris, 1995; un abrégé de Marie Page aux éditions Lito, Champigny-sur-Marne, 1995; une traduction d'Anne Joba et FranceMarie Watkins aux éditions Hachette, 1995; une traduction anonyme aux Éditions de la Fontaine au roy, "Arpège Junior», 1996; une adaptation d'Évelyne Hiest-Lallemand aux éditions Lito, Champigny-sur-Marne, 1997; une adaptation de Chantal Baligand pour les éditions Hemma, Chevron, 2002.

8. Madame Rémy (1872): Petites femmes, Lausanne, H. Mignot, «traduit librement de l'anglais avec l'autorisation de l'auteur»; Madame Rémy (1878): Dictées sur l'orthographe usuelle, les participes et les principales difficultés de la grammaire, Paris, A. Pigoreau ( $3^{\mathrm{e}}$ édition).

9. «I think it was so splendid in father to go as a chaplain when he was too old to be drafted, and not strong enough to be a soldier, said Meg warmly.» (Alcott 1948: 10) Traduction de P.-J. Stahl: «Comme c'est beau à papa d'être parti à l'armée comme médecin, puisqu'il a passé l'âge et qu'il n'aurait plus la force d'être soldat! dit Meg avec enthousiasme.» (Stahl 1880: XXXI, 30)

10. P.-J. Stahl ajoute au titre une note où il s'explique sur son travail d'adaptation: "Cet ouvrage se compose de deux gros volumes dans le texte américain. Je n'ai connu que le premier, par une traduction littérale de M. Lermont. Le second, me dit-on, n'eût pas été possible pour nous. Mais la donnée générale de l'œuvre m'a paru si intéressante que j'ai cru bon de faire pour elle le travail d'adaptation que j'avais fait précédemment pour les Patins d'argent. N'ayant eu à ma disposition que la première moitié de l'ouvrage, j'ai dû, en bien des points, le modifier, l'arranger, pour le conduire à un dénouement qui ne pouvait être celui de l'auteur, puisque je l'ignorais. Le livre, tel qu'il était, n'aurait pu, je le crains, réussir en France; mais la physionomie des caractères des quatre sœurs méritait d'être conservée dans ses lignes principales. J'ai cru pouvoir en faire une lecture agréable et profitable pour nos jeunes lecteurs, et je ne regretterai pas mes efforts si j'y suis parvenu.» (Stahl 1880: XXXI, 24)

11. Rééditions successives: 1923, 1934, 1952 pour la «Bibliothèque Verte» et 1950 pour «Idéal-Bibliothèque».

12. Petites Américaines, Mme Tissier de Mallerais, Hachette, 1935, Meilleurs romans étrangers; Little Women. Les Quatre sœurs Marsch (sic), Henriette Rouillard, Paris, Delagrave, 1936; Les Filles du Dr. March. Traduction nouvelle de Madame H. Giraud. Paris, Pré-aux-Clercs, 1945. Les Quatre sœurs March, Germaine Lalande, Paris, Hazan, 1946.

13. La première édition, parue sous le titre Petites bonnes femmes, les quatre filles du docteur March, portait la mention «Adaptation de R. et A. Prophétie» (Paris, G.P., «Bibliothèque Rouge et or », 34).

14. Exemple, au chapitre 8, «mais son ennemi intérieur, son Apollyon, comme le vieux Bunyan l'appelle dans le voyage du Pèlerin, était toujours prêt à la faire s'irriter, et à vaincre sa résolution » (75) traduisant «but her bosom enemy was always ready to flame up and defeat her» (Alcott 1948: 96).

15. «Madame March, mal portante, ne pouvait les accompagner, mais elle les avait dans la journée recommandées aux soins d'une de ses amies, qu'elles devaient retrouver au bal.» (Alcott 1993: 25; Stahl 1880: XXXI, 89)

16. Jo plaide auprès de sa mère: «- Il est malade, avait-elle dit, et très changé, chez les Gardiner, il m'avait presque fait l'effet d'un petit jeune monsieur, mais je vois bien que ce n'est encore qu'un petit garçon.» (Alcott 1993: 37; Stahl 1880: XXXI, 153)

17. On y lit par exemple qu' « un Américain rougirait de penser à la dot de sa fiancée et [que], s'il lui arrivait de s'en inquiéter et de s'en enquérir publiquement, il ne trouverait plus une fille honorable qui consentît à porter son nom». Et la visiteuse britannique, admirative de tant de vertu, s'exclamerait: «Dieu veuille qu'en vieillissant l'Amérique ne perde pas ces sages principes. » (Alcott 1993: 7879; Stahl 1880: XXXI, 377)

18. Monsieur Laurence appelle Laurie, âgé de seize ans, un «marmot» (Alcott 1993: 168).

19. Ajout de l'adaptateur: "Il y avait évidemment un air de ressemblance entre elle et Margaret, mais Jo était très différente d'aspect.» (Alcott 1988b: 9)

20. Voir, au chapitre 4, sa réponse au reproche de Meg, «Don't use such dreadful expressions »: «I like good, strong words that mean something. » (Alcott 1948: 45)

21. Il faut en outre noter que le choix de cette exclamation n'est peut-être pas fortuit, Louisa May Alcott étant née un Colombus Day, le 29 novembre 1932! 
22. Dans un paragraphe tenant lieu de postface, P.J. Stahl assure que "pas plus qu'un être humain un livre ne voyage impunément» et qu'il faut se résigner à "sacrifier quelque chose aux goûts et aux mœurs» du pays qui adopte le livre (Stahl 1880: XXXII, 344).

23. Cf. l'entrée de son journal pour le $1^{\text {er }}$ novembre 1968: "Girls write to ask who the little women marry, as if that was the only end and aim of a woman's life. I won't marry Jo to Laurie to please any one." (Myerson \& Shealy 1989: 167)

\section{RÉFÉRENCES}

a) Textes ayant servi de base à l'analyse comparative

Alcotт, L. M. (1948) : Little Women, London, J. M. Dent \& Sons Ltd., New York, E.P. Dutton \& Co. Inc.

Alcotт, L. M. (1980): Les quatre filles du Docteur March, adaptation de P.-J. Stahl, Paris, Hachette, "Idéal-Bibliothèque ».

Alcotт, L. M. (1987): Les quatre filles du Docteur March, adaptation de Chantal Baligand, Chevron, Éditions Hemma, «Livre Club Jeunesse».

Alcoтt, L. M. (1988a): Les quatre filles du Docteur March, traduit de l'anglais par Paulette Vielhomme-Callais, Paris, Gallimard, «Folio Junior».

Alcotт, L. M. (1988b): Les quatre filles du Docteur March, adaptation de Rémi Simon, Paris, Nathan, «Bibliothèque des Grands Classiques ».

Alcotт, L. M. (1993) : Les quatre filles du Docteur March, sans indication de traducteur, Tournai, Casterman.

Alcotт, L. M. (1995): Les quatre filles du Docteur March, traduit de l'anglais (États-Unis) par Maud Godoc, Paris, Flammarion, «Castor Poche».

Alcotт, L. M. (2002) : Les quatre filles du Docteur March, adaptation de Chantal Baligand, Chevron, Hemma, «Livre Club jeunesse».

STAHL, P.-J. (1880): Les quatre filles du docteur Marsch (sic). Histoire d'une famille américaine adaptée de L.-L. [sic] Alcott, d'après la traduction de Lermont, Magasin d'éducation et de récréation, vol. XXXI-XXXII.

\section{b) Études et éditions}

Barton, C. H. (1996): Transcendental Wife. The Life of Abigail May Alcott, Lanham, Md., The University Press of America.

Eiselein, G. and A. K. Phillips (2001): "Little Women», in Gregory Eiselein and Anne K. Phillips, eds: The Louisa May Alcott Encyclopedia, Westport, Connecticut/London, Greenwood Press, 178-182.

Elbert, S. (1987): A Hunger for Home: Louisa May Alcott's Place in American Culture, Philadelphia, Temple University Press.

Elbert, S. (1997) : Louisa May Alcott on Race, Sex, and Slavery (edited with an Introduction by), Boston, Northeastern University Press.

Grenier, C. (1993) : «Les couleurs d'un siècle. Un carrefour d'enfances », in Escarpit, Denise et Bernadette Poulou, éd., Paris, Éditions du Sorbier, p. 159-168.

Kerbrat-Orecchioni, C. (1980) : L'Énonciation: De la subjectivité dans le langage, Paris, Armand Colin

Hamon, P. (1984): Texte et idéologie. Valeurs, hiérarchies et évaluations dans l'œuvre littéraire, Paris, PUF.

Holmes Holtz, S. (1995) : "Alcott, Louisa May» in Anita Silvey, ed., Children's Books and Their Creators, Houghton Mifflin Company, Boston/New York, 10-12.

Langland, E. (1983) : "Female Stories of Experience: Alcott's Little Women in Light of Work», in Aвel, Elizabeth, Marianne Hirsch and Elizabeth Langland, ed. : The Voyage In: Fictions of Female Development, Hanover and London, University Press of New England, 112-127. 
Myerson, J. and D. Shealy (1989): The Journals of Louisa May Alcott, with an introduction by Madeleine Stern, Boston/Toronto/London, Little, Brown and Company.

Prince, G. (1978) : «Le discours attributif et le récit», Poétique 35, 305-313.

Reynolds, L. J. (1988): European Revolutions and the American Literary Renaissance, New Haven, Yale University Press.

Shavit, Z. (1986): Poetics of Children's Literature, Athens/London, The University of Georgia Press.

Soriano, M. (1975): «Alcott Louisa Mary (sic)», Guide de littérature pour la jeunesse: courants, problèmes, choix d'auteurs, Paris, Flammarion, 41.

Stern, M. B. (1975): Behind a Mask: The Unknown Thrillers of Louisa May Alcott (edited and with an introduction by), London, W.H. Allen.

Stern, M. B. (1995): Louisa May Alcott, New York, Random House.

Stern, M. B. (1996): The Feminist Alcott; Stories of a woman's power (edited with an introduction by), Boston, Northeastern University.

\section{ANNEXES}

\section{Tableau 1}

\begin{tabular}{|c|c|c|c|c|c|}
\hline LW: 5 & $\mathrm{H}: 10$ & GP : 12 & $\mathrm{~N}: 9-10$ & $\mathrm{~F}: 14$ & CP : 9-10 \\
\hline $\begin{array}{l}\text { Very tall, thin, } \\
\text { and brown }\end{array}$ & $\begin{array}{l}\text { Très grande, } \\
\text { très maigre et } \\
\text { très brune }\end{array}$ & $\begin{array}{l}\text { Très grande, } \\
\text { mince et } \\
\text { brune }\end{array}$ & $\begin{array}{l}\text { Très grande, } \\
\text { mince et } \\
\text { nerveuse, et de } \\
\text { cheveux plus } \\
\text { foncés }\end{array}$ & $\begin{array}{l}\text { Grande, } \\
\text { maigre et } \\
\text { brune }\end{array}$ & $\begin{array}{l}\text { Très élancée et } \\
\text { très brune }\end{array}$ \\
\hline long limbs & $\begin{array}{l}\text { longs } \\
\text { membres }\end{array}$ & $\begin{array}{l}\text { longs } \\
\text { membres }\end{array}$ & $\begin{array}{l}\text { ses longs bras } \\
\text { et ses jambes }\end{array}$ & $\begin{array}{l}\text { bras et jambes } \\
\text { d'une } \\
\text { longueur } \\
\text { interminable }\end{array}$ & $\begin{array}{l}\text { longs } \\
\text { membres }\end{array}$ \\
\hline $\begin{array}{l}\text { decided } \\
\text { mouth }\end{array}$ & $\begin{array}{l}\text { bouche bien } \\
\text { dessinée }\end{array}$ & $\begin{array}{l}\text { bouche } \\
\text { décidée }\end{array}$ & SUPPRESSION & bouche ferme & $\begin{array}{l}\text { bouche } \\
\text { décidée }\end{array}$ \\
\hline comical nose & nez retroussé & nez comique & SUPPRESSION & $\begin{array}{l}\text { drôle de nez } \\
\text { retroussé }\end{array}$ & $\begin{array}{l}\text { drôle de petit } \\
\text { nez }\end{array}$ \\
\hline $\begin{array}{l}\text { sharp grey } \\
\text { eyes }\end{array}$ & yeux gris & $\begin{array}{l}\text { yeux gris } \\
\text { perçants }\end{array}$ & SUPPRESSION & yeux gris & $\begin{array}{l}\text { yeux gris } \\
\text { perçants }\end{array}$ \\
\hline $\begin{array}{l}\text { by turns fierce, } \\
\text { funny, or } \\
\text { thoughtful }\end{array}$ & $\begin{array}{l}\text { tour à tour } \\
\text { pensifs, } \\
\text { moqueurs ou } \\
\text { brillants de } \\
\text { colère }\end{array}$ & $\begin{array}{l}\text { tour à tour } \\
\text { farouches, } \\
\text { amusants, ou } \\
\text { pensifs }\end{array}$ & SUPPRESSION & $\begin{array}{l}\text { à l'expression } \\
\text { tour à tour } \\
\text { féroce, pensive } \\
\text { ou amusée }\end{array}$ & $\begin{array}{l}\text { tour à tour } \\
\text { furieux, } \\
\text { amusés ou } \\
\text { songeurs }\end{array}$ \\
\hline $\begin{array}{l}\text { round } \\
\text { shoulders }\end{array}$ & larges épaules & SUPPRESSION & larges épaules & $\begin{array}{l}\text { épaules bien } \\
\text { découplées }\end{array}$ & épaules rondes \\
\hline $\begin{array}{l}\text { big hands and } \\
\text { feet }\end{array}$ & $\begin{array}{l}\text { de grandes } \\
\text { mains et de } \\
\text { grands pieds }\end{array}$ & SUPPRESSION & $\begin{array}{l}\text { grandes mains } \\
\text { quelque peu } \\
\text { masculines }\end{array}$ & $\begin{array}{l}\text { grandes mains } \\
\text { et grands } \\
\text { pieds }\end{array}$ & $\begin{array}{l}\text { grands pieds } \\
\text { et grandes } \\
\text { mains }\end{array}$ \\
\hline
\end{tabular}


Tableau 2

\begin{tabular}{|c|c|c|c|c|c|c|}
\hline LW: 261 & $\mathrm{H}: 152$ & GP: 159 & C: 146 & $\mathrm{~N}: 157$ & $\mathrm{FJ}: 325$ & $\mathrm{CP}: 276$ \\
\hline $\begin{array}{l}\text { Jo pulled her } \\
\text { hair again } \\
\text { with a } \\
\text { wrathful } \\
\text { tweak }\end{array}$ & $\begin{array}{l}\text { Et Jo passa sa } \\
\text { main dans } \\
\text { ses cheveux } \\
\text { d'un air } \\
\text { désespéré }\end{array}$ & $\begin{array}{l}\text { Et Jo } \\
\text { fourragea } \\
\text { dans ses } \\
\text { cheveux avec } \\
\text { fureur }\end{array}$ & $\begin{array}{l}\text { Et Jo se passa } \\
\text { la main dans } \\
\text { les cheveux } \\
\text { d'un air } \\
\text { désespéré }\end{array}$ & SUPPRESSION & $\begin{array}{l}\text { S'écria Jo qui } \\
\text { se remit à } \\
\text { tirer rageu- } \\
\text { sement sur } \\
\text { ses cheveux } \\
\text { Protesta Jo }\end{array}$ & $\begin{array}{l}\text { qui recom- } \\
\text { mença de } \\
\text { plus belle à } \\
\text { tirer rageu- } \\
\text { sement sur } \\
\text { ses cheveux. }\end{array}$ \\
\hline
\end{tabular}

Tableau 3

\begin{tabular}{|c|c|c|c|c|c|c|}
\hline LW : 97-98 & $\mathrm{H}: 60-61$ & GP: $75-76$ & $C: 55-56$ & $\mathrm{~N}: 76-77$ & $\mathrm{FJ}: 125-26$ & CP : 103-05 \\
\hline $\begin{array}{l}\text { cried Jo, } \\
\text { taking her } \\
\text { by the shoul- } \\
\text { ders, and } \\
\text { looking fierce } \\
\text { enough to } \\
\text { frighten a } \\
\text { much braver } \\
\text { child that } \\
\text { Amy }\end{array}$ & $\begin{array}{l}\text { s'écria Jo en } \\
\text { la prenant } \\
\text { par les } \\
\text { épaules }\end{array}$ & $\begin{array}{l}\text { cria Jo, la } \\
\text { saisissant par } \\
\text { les épaules, } \\
\text { avec une } \\
\text { figure à } \\
\text { épouvanter } \\
\text { plus brave } \\
\text { qu'Amy }\end{array}$ & $\begin{array}{l}\text { s'écria Jo, en } \\
\text { la prenant } \\
\text { par les } \\
\text { épaules et } \\
\text { paraissant } \\
\text { assez en } \\
\text { colère pour } \\
\text { effrayer une } \\
\text { enfant } \\
\text { beaucoup } \\
\text { plus brave } \\
\text { qu'Amy }\end{array}$ & SUPPRESSION & $\begin{array}{l}\text { Jo la saisit } \\
\text { par les } \\
\text { épaules avec } \\
\text { fureur [...] } \\
\text { s'écria-t-elle } \\
\text { avec une } \\
\text { expression } \\
\text { qui aurait } \\
\text { fait peur à } \\
\text { quelqu'un } \\
\text { de beaucoup } \\
\text { plus cou- } \\
\text { rageux } \\
\text { qu'Amy }\end{array}$ & $\begin{array}{l}\text { s'écria Jo en } \\
\text { l'empoignant } \\
\text { par les } \\
\text { épaules d'un } \\
\text { air menaçant } \\
\text { qui aurait } \\
\text { effrayé plus } \\
\text { courageux } \\
\text { qu'Amy. }\end{array}$ \\
\hline $\begin{array}{l}\text { And Jo gave } \\
\text { her a light } \\
\text { shake }\end{array}$ & SUPPRESSION & $\begin{array}{l}\text { Dit Jo en la } \\
\text { secouant }\end{array}$ & $\begin{array}{l}\text { Et Jo la } \\
\text { secoua } \\
\text { légèrement }\end{array}$ & SUPPRESSION & $\begin{array}{l}\text { Dit Jo en la } \\
\text { secouant un } \\
\text { peu }\end{array}$ & $\begin{array}{l}\text { Dit Jo en la } \\
\text { secouant un } \\
\text { peu }\end{array}$ \\
\hline $\begin{array}{l}\text { said Jo, } \\
\text { turning very } \\
\text { pale, while } \\
\text { her eyes } \\
\text { kindled and } \\
\text { her hands } \\
\text { clutched } \\
\text { Amy ner- } \\
\text { vously }\end{array}$ & $\begin{array}{l}\text { demanda Jo } \\
\text { qui serrait } \\
\text { nerveuse- } \\
\text { ment Amy }\end{array}$ & $\begin{array}{l}\text { dit Jo, deve- } \\
\text { nant toute } \\
\text { pâle, mais les } \\
\text { yeux étince- } \\
\text { lants, et } \\
\text { serrant } \\
\text { nerveuse- } \\
\text { ment les } \\
\text { épaules } \\
\text { d'Amy. }\end{array}$ & $\begin{array}{l}\text { demanda Jo, } \\
\text { qui serrait } \\
\text { nerveuse- } \\
\text { ment Amy }\end{array}$ & SUPPRESSION & $\begin{array}{l}\text { les mains de } \\
\text { Jo se crispè- } \\
\text { rent nerveu- } \\
\text { sement sur } \\
\text { les épaules } \\
\text { de sa sœur. } \\
\text { [...] Elle } \\
\text { était devenue } \\
\text { très pâle et } \\
\text { ses yeux } \\
\text { lançaient } \\
\text { des éclairs. }\end{array}$ & $\begin{array}{l}\text { demanda Jo } \\
\text { tandis que } \\
\text { ses mains se } \\
\text { crispaient } \\
\text { nerveuse- } \\
\text { ment sur sa } \\
\text { sœur. Elle } \\
\text { était devenue } \\
\text { livide et ses } \\
\text { yeux lan- } \\
\text { çaient des } \\
\text { éclairs. }\end{array}$ \\
\hline $\begin{array}{l}\text { for Jo's hot } \\
\text { temper } \\
\text { mastered } \\
\text { her; and she } \\
\text { shook Amy } \\
\text { till her teeth } \\
\text { chattered in } \\
\text { her head, } \\
\text { crying, in a } \\
\text { passion of } \\
\text { grief and } \\
\text { anger }\end{array}$ & $\begin{array}{l}\text { Jo, qui ne } \\
\text { pouvait } \\
\text { maîtriser sa } \\
\text { colère, la } \\
\text { secoua si } \\
\text { violemment } \\
\text { qu'Amy en } \\
\text { perdit la } \\
\text { respiration. }\end{array}$ & $\begin{array}{l}\text { car Jo la } \\
\text { secoua à la } \\
\text { faire claquer } \\
\text { des dents. Et } \\
\text { dans un } \\
\text { paroxysme } \\
\text { de chagrin et } \\
\text { de fureur, } \\
\text { elle cria }\end{array}$ & \begin{tabular}{|l|} 
car Jo, qui ne \\
pouvait \\
maîtriser sa \\
colère, la \\
secouait si \\
violemment \\
qu'Amy en \\
perdait la \\
respiration
\end{tabular} & $\begin{array}{l}\text { cria Jo d'un } \\
\text { ton à faire } \\
\text { peur et en la } \\
\text { secouant } \\
\text { comme un } \\
\text { prunier }\end{array}$ & $\begin{array}{l}\text { car la nature } \\
\text { violente de } \\
\text { Jo prit le } \\
\text { dessus, et elle } \\
\text { se mit à } \\
\text { secouer sa } \\
\text { sœur jusqu'à } \\
\text { ce que ses } \\
\text { dents s'entre- } \\
\text { choquent. } \\
\text { [...] cria-t- } \\
\text { elle, folle de } \\
\text { rage et de } \\
\text { chagrin. }\end{array}$ & $\begin{array}{l}\text { car Jo laissa } \\
\text { éclater sa } \\
\text { colère et } \\
\text { secoua sa } \\
\text { sœur jusqu'à } \\
\text { ce que ses } \\
\text { dents s'entre- } \\
\text { choquent. } \\
{[\ldots]} \\
\text { Explosa-t- } \\
\text { elle, folle de } \\
\text { rage }\end{array}$ \\
\hline
\end{tabular}


Tableau 3 (suite)

\begin{tabular}{|c|c|c|c|c|c|c|}
\hline LW : 97-98 & $\mathrm{H}: 60-61$ & GP : 75-76 & $C: 55-56$ & $\mathrm{~N}: 76-77$ & FJ : $125-26$ & CP : 103-05 \\
\hline $\begin{array}{l}\text { but Jo was } \\
\text { quite beside } \\
\text { herself and } \\
\text { with a part- } \\
\text { ing box on } \\
\text { her sister's } \\
\text { ear, she } \\
\text { rushed out } \\
\text { of the room } \\
\text { up to the old } \\
\text { sofa, and } \\
\text { finished her } \\
\text { fight alone }\end{array}$ & $\begin{array}{l}\text { mais celle-ci } \\
\text { était en } \\
\text { colère; elle } \\
\text { s'enfuit au } \\
\text { grenier pour } \\
\text { y cacher son } \\
\text { chagrin. }\end{array}$ & $\begin{array}{l}\text { Jo était hors } \\
\text { d'elle. Admi- } \\
\text { nistrant à } \\
\text { Amy une } \\
\text { maîtresse } \\
\text { gifle, elle se } \\
\text { précipita } \\
\text { hors de la } \\
\text { pièce. }\end{array}$ & $\begin{array}{l}\text { mais celle-ci } \\
\text { était tout à } \\
\text { fait en colère, } \\
\text { et après avoir } \\
\text { donné une } \\
\text { dernière tape } \\
\text { à Amy ... } \\
\text { pour y } \\
\text { cacher son } \\
\text { chagrin }\end{array}$ & $\begin{array}{l}\text { Jo, pâle de } \\
\text { colère et des } \\
\text { larmes de } \\
\text { rage dans les } \\
\text { yeux, levait la } \\
\text { main sur sa } \\
\text { sœur! Un } \\
\text { puissant ré- } \\
\text { flexe l'empê- } \\
\text { cha d'abattre } \\
\underline{\text { sa main (et }} \\
\underline{\text { ce fut bien }} \\
\underline{\text { heureux }} \\
\text { pour Amy, } \\
\underline{\text { car Jo avait }} \\
\underline{\text { facilement la }} \\
\underline{\text { force d'un }} \\
\text { garçon de } \\
\underline{\text { son âge!). }}\end{array}$ & $\begin{array}{l}\text { mais celle-ci } \\
\text { était hors } \\
\text { d'elle, et, } \\
\text { après avoir } \\
\text { flanqué une } \\
\text { dernière } \\
\text { taloche à } \\
\text { Amy en guise } \\
\text { d'adieu... } \\
\text { [...] } \\
\text { pour rumi- } \\
\text { ner sa colère }\end{array}$ & $\begin{array}{l}\text { mais celle-ci } \\
\text { était hors } \\
\text { d'elle et, } \\
\text { après avoir } \\
\text { gratifié sa } \\
\text { sœur d'une } \\
\text { dernière } \\
\text { gifle, elle } \\
\text { sortit en } \\
\text { trombe de la } \\
\text { pièce et se } \\
\text { précipita au } \\
\text { grenier pour } \\
\text { vider sa } \\
\text { colère, seule } \\
\text { sur le vieux } \\
\text { sofa. }\end{array}$ \\
\hline $\begin{array}{l}\text { putting her } \\
\text { whole heart } \\
\text { into her } \\
\text { work, hoping } \\
\text { to make } \\
\text { something } \\
\text { good enough } \\
\text { to print }\end{array}$ & $\begin{array}{l}\text { la pauvre Jo } \\
\text { s'était dit } \\
\text { qu'ainsi, par } \\
\text { son travail, si } \\
\text { elle réussis- } \\
\text { sait à faire, } \\
\text { elle pourrait } \\
\text { venir en aide } \\
\text { à sa mère. }\end{array}$ & $\begin{array}{l}\text { mais Jo y } \\
\text { avait travaillé } \\
\text { avec patience, } \\
\text { espérant } \\
\text { faire quelque } \\
\text { chose d'assez } \\
\text { bon pour } \\
\text { l'impression. }\end{array}$ & $\begin{array}{l}\text { Jo espérait } \\
\text { avoir fait } \\
\text { quelque } \\
\text { chose d'assez } \\
\text { bien pour } \\
\text { être imprimé } \\
\text { dans un } \\
\text { journal très } \\
\text { aimé des } \\
\underline{\text { bébés. La }} \\
\text { pauvre Jo } \\
\text { s'était dit } \\
\text { qu'ainsi, par } \\
\text { son travail, si } \\
\text { elle réussis- } \\
\text { sait à faire, } \\
\text { elle pourrait } \\
\text { venir en aide } \\
\text { à sa mère. }\end{array}$ & $\begin{array}{l}\text { contes qu'elle } \\
\text { espérait } \\
\text { vivement } \\
\text { publier un } \\
\text { jour }\end{array}$ & $\begin{array}{l}\text { et y avait mis } \\
\text { tout son } \\
\text { cœur dans } \\
\text { l'espoir qu'ils } \\
\text { seraient assez } \\
\text { bons pour } \\
\text { être publiés } \\
\text { un jour }\end{array}$ & $\begin{array}{l}\text { en y mettant } \\
\text { tout son } \\
\text { cœur dans } \\
\text { l'espoir qu'ils } \\
\text { seraient assez } \\
\text { bons pour } \\
\text { être publiés. }\end{array}$ \\
\hline $\begin{array}{l}\text { To Jo it was } \\
\text { a dreadful } \\
\text { calamity, } \\
\text { and she felt } \\
\text { that it never } \\
\text { could be } \\
\text { made up to } \\
\text { her }\end{array}$ & $\begin{array}{l}\text { C'était tout } \\
\text { un rêve } \\
\text { innocent } \\
\text { détruit. }\end{array}$ & $\begin{array}{l}\text { Pour Jo, } \\
\text { c'était une } \\
\text { terrible } \\
\text { calamité }\end{array}$ & $\begin{array}{l}\text { C'était tout } \\
\text { un rêve } \\
\text { innocent } \\
\text { détruit. }\end{array}$ & SUPPRESSION & $\begin{array}{l}\text { Était pour Jo } \\
\text { une terrible } \\
\text { catastrophe } \\
\text { et elle avait } \\
\text { le sentiment } \\
\text { d'une perte } \\
\text { irréparable. }\end{array}$ & $\begin{array}{l}\text { Un désastre } \\
\text { sans nom } \\
\text { aux yeux de } \\
\text { Jo et elle } \\
\text { avait l'im- } \\
\text { pression } \\
\text { d'avoir subi } \\
\text { un malheur } \\
\text { irréparable. }\end{array}$ \\
\hline
\end{tabular}


Tableau 4

\begin{tabular}{|c|c|c|c|c|c|c|}
\hline LW : 101 & $\mathrm{H}: 62-63$ & GP: $77-78$ & $\mathrm{C}: 58$ & $\mathrm{~N}: 79$ & FJ: $130-31$ & CP : 107-08 \\
\hline $\begin{array}{l}\text { But Jo never } \\
\text { turned, and } \\
\text { went slowly } \\
\text { zigzagging } \\
\text { down the } \\
\text { river, taking } \\
\text { a bitter, } \\
\text { unhappy sort } \\
\text { of satisfac- } \\
\text { tion in her } \\
\text { sister's } \\
\text { troubles. She } \\
\text { had cher- } \\
\text { ished her } \\
\text { anger till it } \\
\text { grew strong, } \\
\text { and took } \\
\text { possession } \\
\text { of her, as } \\
\text { evil thoughts } \\
\text { and feelings } \\
\text { always do, } \\
\text { unless cast } \\
\text { at once. }\end{array}$ & SUPPRESSION & SUPPRESSION & SUPPRESSION & SUPPRESSION & $\begin{array}{l}\text { Elle partit } \\
\text { sans se } \\
\text { retourner et } \\
\text { se mit à } \\
\text { zizaguer sur } \\
\text { la glace en se } \\
\text { disant avec } \\
\text { une joie } \\
\text { mauvaise } \\
\text { que sa sœur } \\
\text { n'avait qu'à } \\
\text { se dépêtrer } \\
\text { avec ses } \\
\text { patins. Jo } \\
\text { avait entre- } \\
\text { tenu sa } \\
\text { colère et } \\
\text { l'avait laissé } \\
\text { grandir } \\
\text { jusqu'à ce } \\
\text { qu'elle l'en- } \\
\text { vahisse } \\
\text { complète- } \\
\text { ment, ce que } \\
\text { font toujours } \\
\text { les pensées et } \\
\text { les senti- } \\
\text { ments néga- } \\
\text { tifs lorsqu'on } \\
\text { ne les rejette } \\
\text { pas immé- } \\
\text { diatement. }\end{array}$ & $\begin{array}{l}\text { Mais elle ne } \\
\text { se retourna } \\
\text { pas et se mit } \\
\text { à zigzaguer } \\
\text { avec lenteur } \\
\text { sur la rivière. } \\
\text { Se délectant } \\
\text { avec une joie } \\
\text { mauvaise des } \\
\text { petits tracas } \\
\text { de sa sœur. } \\
\text { Elle avait } \\
\text { nourri sa } \\
\text { colère qui } \\
\text { avait fini par } \\
\text { prendre } \\
\text { possession } \\
\text { d'elle-même, } \\
\text { comme } \\
\text { chaque fois } \\
\text { qu'on ne la } \\
\text { bannit pas } \\
\text { d'emblée. }\end{array}$ \\
\hline $\begin{array}{l}\text { And the little } \\
\text { demon she } \\
\text { was har- } \\
\text { bouring said } \\
\text { in her ear: } \\
\text { 'No matter } \\
\text { whether she } \\
\text { hears or not, } \\
\text { let her take } \\
\text { care of } \\
\text { herself'. }\end{array}$ & SUPPRESSION & $\begin{array}{l}\text { Et le petit } \\
\text { démon } \\
\text { qu'elle logeait } \\
\text { dans son } \\
\text { cœur, son } \\
\text { Apollyon, lui } \\
\text { dit à l'oreille: } \\
\text { "Ça ne fait } \\
\text { rien si elle } \\
\text { n'a pas } \\
\text { entendu: } \\
\text { c'est à elle de } \\
\text { prendre ses } \\
\text { précau- } \\
\text { tions." }\end{array}$ & $\begin{array}{l}\text { Et le petit } \\
\text { démon de la } \\
\text { rancune } \\
\text { qu'elle abri- } \\
\text { tait dans son } \\
\text { cœur lui dit } \\
\text { à l'oreille: } \\
\text { "Elle m'a ôté } \\
\text { le droit de } \\
\text { prendre soin } \\
\text { d'elle! }\end{array}$ & $\begin{array}{l}\text { «A-t-elle } \\
\text { entendu? se } \\
\text { demanda Jo. } \\
\text { Oh! et puis } \\
\text { zut! Elle n'a } \\
\text { qu'à s'occu- } \\
\text { per d'elle- } \\
\text { même.» }\end{array}$ & $\begin{array}{l}\text { Et le petit } \\
\text { démon qui } \\
\text { l'habitait lui } \\
\text { souffla à } \\
\text { l'oreille: } \\
\text { "Tant pis si } \\
\text { elle n'a pas } \\
\text { entendu, elle } \\
\text { peut bien se } \\
\text { débrouiller } \\
\text { toute seule. " }\end{array}$ & $\begin{array}{l}\text { Et le petit } \\
\text { démon qui } \\
\text { l'habitait lui } \\
\text { souffla à } \\
\text { l'oreille: } \\
\text { Qu'importe } \\
\text { qu'elle ait } \\
\text { entendu ou } \\
\text { non, elle n'a } \\
\text { qu'à se } \\
\text { débrouiller } \\
\text { toute seule. } \\
\text { For a minute }\end{array}$ \\
\hline $\begin{array}{l}\text { Jo stood still, } \\
\text { with a } \\
\text { strange } \\
\text { feeling at her } \\
\text { heart; then } \\
\text { she resolved } \\
\text { to go on, but } \\
\text { something }\end{array}$ & $\begin{array}{l}\text { Cependant, } \\
\text { en voyant } \\
\text { Amy qui, le } \\
\text { nez au vent, } \\
\text { patinait vers } \\
\text { le milieu de } \\
\text { la rivière, Jo } \\
\text { eut un }\end{array}$ & $\begin{array}{l}\text { Jo résolut de } \\
\text { continuer; } \\
\text { malgré tout, } \\
\text { elle se re- } \\
\text { tourna, juste } \\
\text { pour voir } \\
\text { Amy lever les } \\
\text { bras au ciel, }\end{array}$ & $\begin{array}{l}\text { Pendant une } \\
\text { minute, Jo, } \\
\text { inquiète sans } \\
\text { vouloir le } \\
\text { paraître, resta } \\
\text { immobile; } \\
\text { elle hésita un } \\
\text { instant, puis, }\end{array}$ & $\begin{array}{l}\text { Jo eut un } \\
\text { mouvement } \\
\text { d'inquiétude. } \\
\text { L'instant } \\
\text { d'après, la } \\
\text { glace cra- } \\
\text { quait et Amy, } \\
\text { avec un cri }\end{array}$ & $\begin{array}{l}\text { Prise d'un } \\
\text { curieux } \\
\text { malaise, Jo } \\
\text { s'arrêta un } \\
\text { moment; elle } \\
\text { allait repartir } \\
\text { lorsque } \\
\text { quelque }\end{array}$ & $\begin{array}{l}\text { Jo s'immobi- } \\
\text { lisa sur la } \\
\text { glace, en } \\
\text { proie à une } \\
\text { curieuse } \\
\text { oppression. } \\
\text { Elle s'apprê- } \\
\text { tait à repartir }\end{array}$ \\
\hline
\end{tabular}


Tableau 4 (suite)

\begin{tabular}{|c|c|c|c|c|c|c|}
\hline LW: & $H: 62-63$ & GP: $77-78$ & $: 58$ & $N: 79$ & FJ : $130-31$ & CP : 107-08 \\
\hline $\begin{array}{l}\text { held and } \\
\text { turned her } \\
\text { round, just } \\
\text { in time to see } \\
\text { Amy throw } \\
\text { up her hands } \\
\text { and go } \\
\text { down, with } \\
\text { the sudden } \\
\text { crash of } \\
\text { rotten ice, } \\
\text { the splash of } \\
\text { water, and a } \\
\text { cry that } \\
\text { made Jo's } \\
\text { heart stand } \\
\text { still with fear. } \\
\text { She tried to } \\
\text { call Laurie } \\
\text { but her voice } \\
\text { was gone; } \\
\text { she tried to } \\
\text { rush forward, } \\
\text { but her feet } \\
\text { seemed to } \\
\text { have no } \\
\text { strenghth in } \\
\text { them; and } \\
\text { for a second, } \\
\text { she could } \\
\text { only stand } \\
\text { motionless, } \\
\text { staring, with } \\
\text { a terror- } \\
\text { stricken face, } \\
\text { at the little } \\
\text { blue hood } \\
\text { above the } \\
\text { black water }\end{array}$ & $\begin{array}{l}\text { moment } \\
\text { d'inquiétude. } \\
\text { L'instant } \\
\text { d'après, la } \\
\text { glace cra- } \\
\text { quait et Amy, } \\
\text { avec un cri } \\
\text { aigu, s'en- } \\
\text { fonça dans } \\
\text { l'eau glacée. } \\
\text { Jo resta } \\
\text { pétrifiée: elle } \\
\text { essaya d'ap- } \\
\text { peler au } \\
\text { secours, mais } \\
\text { pa s un son } \\
\text { ne sortit de } \\
\text { sa gorge et } \\
\text { pendant } \\
\text { quelques } \\
\text { horribles } \\
\text { secondes elle } \\
\text { ne vit plus } \\
\text { que le petit } \\
\text { capuchon } \\
\text { bleu qui se } \\
\text { débattait au } \\
\text { milieu des } \\
\text { morceaux de } \\
\text { glace. }\end{array}$ & $\begin{array}{l}\text { et s'enfoncer } \\
\text { avec un } \\
\text { craquement } \\
\text { de glace } \\
\text { rompue et } \\
\text { un cri qui fit } \\
\text { arrêter de } \\
\text { terreur le } \\
\text { cœur de Jo. } \\
\text { Pendant une } \\
\text { seconde, elle } \\
\text { ne peut que } \\
\text { demeurer } \\
\text { immobile, en } \\
\text { regardant } \\
\text { avec épou- } \\
\text { vante le petit } \\
\text { capuchon } \\
\text { bleu au- } \\
\text { dessus de } \\
\text { l'eau noire. }\end{array}$ & 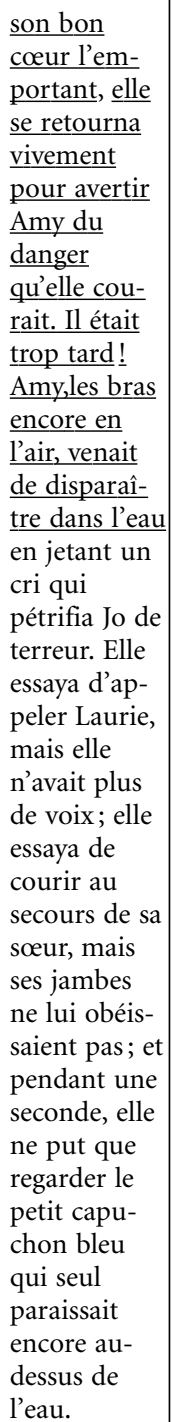 & $\begin{array}{l}\text { aigu, s'en- } \\
\text { fonça dans } \\
\text { l'eau glacée. } \\
\text { Jo resta } \\
\text { pétrifiée: elle } \\
\text { essaya d'ap- } \\
\text { peler au } \\
\text { secours, mais } \\
\text { pas un son } \\
\text { ne sortit de } \\
\text { sa gorge et } \\
\text { pendant } \\
\text { quelques } \\
\text { horribles } \\
\text { secondes elle } \\
\text { ne vit plus } \\
\text { que le petit } \\
\text { capuchon } \\
\text { bleu qui se } \\
\text { débattait au } \\
\text { milieu des } \\
\text { morceaux de } \\
\text { glace. }\end{array}$ & $\begin{array}{l}\text { chose la } \\
\text { retint. Elle } \\
\text { entendit un } \\
\text { fracas de } \\
\text { glace brisée } \\
\text { et un cri qui } \\
\text { lui glaça le } \\
\text { sang. Jo } \\
\text { voulut appe- } \\
\text { ler Laurie, } \\
\text { mais elle } \\
\text { n'avait plus } \\
\text { de voix. Elle } \\
\text { essaya de } \\
\text { courir, mais } \\
\text { ses jambes } \\
\text { ne lui obéis- } \\
\text { saient plus. } \\
\text { Pendant une } \\
\text { seconde, elle } \\
\text { ne put que } \\
\text { regarder avec } \\
\text { des yeux } \\
\text { dilatés le } \\
\text { petit bonnet } \\
\text { bleu qui } \\
\text { surnageait } \\
\text { sur l'eau } \\
\text { noire. }\end{array}$ & $\begin{array}{l}\text { quand un } \\
\text { pressenti- } \\
\text { ment la } \\
\text { retint: au } \\
\text { moment } \\
\text { même où elle } \\
\text { se retournait, } \\
\text { elle vit Amy, } \\
\text { les bras au } \\
\text { ciel, être } \\
\text { happée par la } \\
\text { rivière dans } \\
\text { un craque- } \\
\text { ment sinistre, } \\
\text { avec un cri } \\
\text { qui lui glaça } \\
\text { le sang. Jo } \\
\text { essaya d'ap- } \\
\text { peler Laurie, } \\
\text { mais aucun } \\
\text { son ne sortit } \\
\text { de sa gorge. } \\
\text { Quand elle } \\
\text { voulut se } \\
\text { précipiter au } \\
\text { secours de sa } \\
\text { sœur, ses } \\
\text { jambes, en } \\
\text { coton, refu- } \\
\text { sèrent de lui } \\
\text { obéir et elle } \\
\text { ne put que } \\
\text { contempler, } \\
\text { les yeux } \\
\text { écarquillés } \\
\text { de terreur, le } \\
\text { petit capu- } \\
\text { chon bleu } \\
\text { qui surna- } \\
\text { geait sur } \\
\text { l'eau sombre. }\end{array}$ \\
\hline
\end{tabular}

Tableau 5

\begin{tabular}{|l|l|l|l|l|l|}
\hline LW : 47 & H: 37 & GP: 45 & N $: 53$ & FJ: 65 & CP: 55 \\
\hline $\begin{array}{l}\text { something in } \\
\text { her comical } \\
\text { face and blunt } \\
\text { manners struck } \\
\text { the old lady's } \\
\text { fancy. }\end{array}$ & $\begin{array}{l}\text { l'originalité de } \\
\text { la jeune fille } \\
\text { lui plut. }\end{array}$ & $\begin{array}{l}\text { sa drôle de } \\
\text { figure et ses } \\
\text { manières } \\
\text { brusques lui } \\
\text { plurent. }\end{array}$ & $\begin{array}{l}\text { sa nièce Jo dont } \\
\text { le visage franc } \\
\text { et les manières } \\
\text { brusques lui } \\
\text { avaient plu. }\end{array}$ & $\begin{array}{l}\text { sa drôle de } \\
\text { frimousse et } \\
\text { ses manières } \\
\text { directes lui } \\
\text { avaient plu. }\end{array}$ & $\begin{array}{l}\text { son } \underline{\text { minois }} \\
\text { facétieux et ses } \\
\text { manières un } \\
\text { peu brusques } \\
\text { lui avaient plu. }\end{array}$ \\
\hline
\end{tabular}


Tableau 6

\begin{tabular}{|c|c|c|c|c|c|c|}
\hline LW: 159 & $\mathrm{H}$ & GP: 101 & $\mathrm{C}: 75$ & $\mathrm{~N}: 94$ & FJ : 203 & $\mathrm{CP}: 172$ \\
\hline $\begin{array}{l}\text { Kate looked } \\
\text { rather } \\
\text { amazed at } \\
\text { Jo's proceed- } \\
\text { ings, [...]. } \\
\text { But after } \\
\text { putting up } \\
\text { her glass to } \\
\text { examine the } \\
\text { queer girl } \\
\text { several times, } \\
\text { Miss Kate } \\
\text { decided that } \\
\text { she was 'odd, } \\
\text { but rather } \\
\text { clever', and } \\
\text { smiled upon } \\
\text { her from } \\
\text { afar. }\end{array}$ & SUPPRESSION & $\begin{array}{l}\begin{array}{l}\text { Kate eut l'air } \\
\text { plutôt ahurie } \\
\text { devant les } \\
\text { manières de }\end{array} \\
\text { Jo [...] Mais } \\
\text { après avoir } \\
\text { braqué son } \\
\text { face-à-main } \\
\text { pour } \\
\text { examiner } \\
\text { cette fille } \\
\text { extraordi- } \\
\underline{\text { naire, à }} \\
\text { plusieurs } \\
\text { reprises, Miss } \\
\text { Kate décida } \\
\text { qu'elle était } \\
\text { bizarre, mais } \\
\text { plutôt } \\
\text { sympathique, } \\
\text { et lui sourit } \\
\text { de loin. }\end{array}$ & 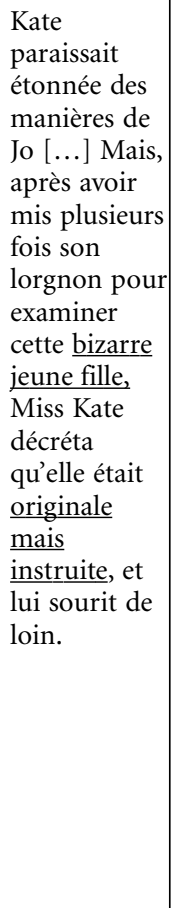 & $\begin{array}{l}\text { Kate, qui } \\
\text { avait d'abord } \\
\text { été étonnée } \\
\text { des façons de } \\
\text { Jo }[\ldots] \\
\text { regarda } \\
\text { longuement } \\
\text { la jeune fille } \\
\text { à travers son } \\
\text { face à main } \\
\text { puis décida } \\
\text { qu'elle était } \\
\text { bizarre mais } \\
\text { pas bête, et } \\
\text { lui fit un } \\
\text { sourire }\end{array}$ & $\begin{array}{l}\text { Kate semblait } \\
\text { passablement } \\
\text { déconcertée } \\
\text { par le com- } \\
\text { portement } \\
\text { de Jo [...] } \\
\text { Mais quand } \\
\text { elle eut pris } \\
\text { plusieurs fois } \\
\text { son lorgnon } \\
\text { pour } \\
\text { examiner } \\
\text { cette } \\
\text { "étrange } \\
\text { créature», } \\
\text { Miss Kate } \\
\text { finit par } \\
\text { conclure } \\
\text { qu'elle était } \\
\text { «bizarre, } \\
\text { mais plutôt } \\
\text { intelligente }, \\
\text { et, de sa } \\
\text { place, lui } \\
\text { adressa } \\
\text { quelques } \\
\text { sourires } \\
\text { distants. }\end{array}$ & $\begin{array}{l}\text { Kate } \\
\text { paraissait } \\
\text { plutôt } \\
\text { déconcertée } \\
\text { par le } \\
\text { comporte- } \\
\text { ment de Jo } \\
{[\ldots] \text { Mais, }} \\
\text { après avoir } \\
\text { dévisagé à } \\
\text { plusieurs } \\
\text { reprises cet } \\
\text { étrange } \\
\text { phénomène } \\
\text { derrière son } \\
\text { face-à-main, } \\
\text { Miss Kate } \\
\text { décréta que } \\
\text { cette jeune } \\
\text { fille était } \\
\text { «certes } \\
\text { bizarre, mais } \\
\text { plutôt } \\
\underline{\text { intelligente }} \\
\text { et lui adressa } \\
\text { quelques } \\
\text { vagues sou- } \\
\text { rires de loin. }\end{array}$ \\
\hline
\end{tabular}

Tableau 7

\begin{tabular}{|c|c|c|c|c|c|c|}
\hline LW: 174 & $\mathrm{H}: 162$ & GP: 169 & $C: 163$ & $\mathrm{~N}: 167$ & $\mathrm{FJ}: 340$ & $\mathrm{CP}: 289$ \\
\hline $\begin{array}{l}\text { If I was a } \\
\text { boy, we'd } \\
\text { run away } \\
\text { together, } \\
\text { and have a } \\
\text { capital time; } \\
\text { but as I am } \\
\text { a miserable } \\
\text { girl I must } \\
\text { be proper, } \\
\text { and stop at } \\
\text { home. }\end{array}$ & $\begin{array}{l}\text { Si j'étais un } \\
\text { garçon, je } \\
\text { dirais oui } \\
\text { tout de suite, } \\
\text { mais je ne } \\
\text { suis qu'une } \\
\text { pauvre fille, } \\
\text { et je dois } \\
\text { rester } \\
\text { sagement au } \\
\text { coin de mon } \\
\text { feu. }\end{array}$ & $\begin{array}{l}\text { SUPPRES- } \\
\text { SION : JO } \\
\text { DISSUADE } \\
\text { IMMÉDIATE- } \\
\text { MENT } \\
\text { LAURIE: } \\
\text { Voyons, } \\
\text { réfléchissez à } \\
\text { vos propres } \\
\text { torts. }\end{array}$ & $\begin{array}{l}\text { Ah, si j'étais } \\
\text { un garçon, } \\
\text { s'écria-t-elle! } \\
\text { Mais non, je } \\
\text { ne suis } \\
\text { qu'une fille, } \\
\text { une malheu- } \\
\text { reuse et } \\
\text { déplorable } \\
\text { fille! Il faut } \\
\text { malgré tout, } \\
\text { Laurie, que je } \\
\text { me conduise } \\
\text { comme une } \\
\text { honnête et } \\
\text { convenable } \\
\text { demoiselle; } \\
\text { et, par suite, } \\
\text { que je reste à } \\
\text { la maison. } \\
\text { Sous l'aile } \\
\text { même de ma } \\
\text { mère. Tout ce } \\
\text { qui ne serait } \\
\text { pas cela serait } \\
\text { démence et } \\
\text { insanité... }\end{array}$ & $\begin{array}{l}\text { Si j'étais un } \\
\text { garçon, fit- } \\
\text { elle, je dirais } \\
\text { oui tout de } \\
\text { suite. Mais } \\
\text { hélas, je ne } \\
\text { suis qu'une } \\
\text { fille, comme } \\
\text { tu le sais sans } \\
\text { doute! C'est } \\
\text { un beau } \\
\text { projet, mais } \\
\text { irréalisable, } \\
\text { malheureuse- } \\
\text { ment. }\end{array}$ & $\begin{array}{l}\text { Si j'étais un } \\
\text { garçon, nous } \\
\text { pourrions } \\
\text { nous sauver } \\
\text { tous les deux } \\
\text { et passer des } \\
\text { moments } \\
\text { formidables; } \\
\text { mais comme } \\
\text { je ne suis } \\
\text { qu'une } \\
\text { misérable } \\
\text { fille, je suis } \\
\text { obligée de } \\
\text { me conduire } \\
\text { convenable- } \\
\text { ment et de } \\
\text { rentrer chez } \\
\text { moi. }\end{array}$ & $\begin{array}{l}\text { Si j'étais un } \\
\text { garçon, on } \\
\text { s'enfuirait } \\
\text { tous les deux } \\
\text { et on } \\
\text { s'amuserait } \\
\text { comme des } \\
\text { fous. Mais je } \\
\text { ne suis } \\
\text { qu'une } \\
\text { misérable } \\
\text { fille, alors je } \\
\text { dois bien me } \\
\text { conduire et } \\
\text { rester à la } \\
\text { maison. }\end{array}$ \\
\hline
\end{tabular}


Tableau 8

\begin{tabular}{|c|c|c|c|c|c|c|}
\hline LW : 287 & $\mathrm{H}: 173$ & GP : 177 & C: 177 & $\mathrm{~N}: 177$ & FJ : 356 & CP : 302 \\
\hline $\begin{array}{l}\text { I rather miss } \\
\text { my wild girl; } \\
\text { but if I get a } \\
\text { strong, } \\
\text { helpful, } \\
\text { tender- } \\
\text { hearted } \\
\text { woman in } \\
\text { her place, I } \\
\text { shall feel } \\
\text { quite satis- } \\
\text { fied. I don't } \\
\text { know if the } \\
\text { shearing } \\
\text { sobered our } \\
\text { black sheep, } \\
\text { but I do } \\
\text { know that in } \\
\text { all Washing- } \\
\text { ton I } \\
\text { couldn't find } \\
\text { anything } \\
\text { beautiful } \\
\text { enough to be } \\
\text { bought with } \\
\text { the five-and- } \\
\text { twenty } \\
\text { dollars which } \\
\text { my good girl } \\
\text { sent me. }\end{array}$ & $\begin{array}{l}\text { J'aimais } \\
\text { certes ma } \\
\text { fille sauvage, } \\
\text { mais je crois } \\
\text { que si j'ai à } \\
\text { la place une } \\
\text { jeune fille } \\
\text { tendre, } \\
\text { dévouée et } \\
\text { civilisée, } \\
\text { j'aurai gagné } \\
\text { au change. Je } \\
\text { sais déjà que } \\
\text { dans tout } \\
\text { Washington, } \\
\text { je n'aurais } \\
\text { rien pu } \\
\text { trouver qui } \\
\text { valût les } \\
\text { vingt-cinq } \\
\text { dollars que } \\
\text { cette chère } \\
\text { tête ronde } \\
\text { m'a envoyés. }\end{array}$ & $\begin{array}{l}\text { Je ne sais si } \\
\text { c'est d'être } \\
\text { tondu qui a } \\
\text { ainsi calmé } \\
\underline{\text { mon vieux }} \\
\underline{\text { mouton }} \\
\underline{\text { noir; mais ce }} \\
\text { que je sais, } \\
\text { c'est que je } \\
\text { n'ai rien } \\
\text { trouvé d'as- } \\
\text { sez beau } \\
\text { dans tout } \\
\text { Washington, } \\
\text { pour être } \\
\text { acheté avec } \\
\text { les vingt-cinq } \\
\text { dollars que } \\
\text { ma bonne } \\
\text { fille m'a } \\
\text { envoyés. }\end{array}$ & $\begin{array}{l}\text { J'aimais } \\
\text { certes ma } \\
\text { fille sauvage, } \\
\text { mais je crois } \\
\text { que, si j'ai } \\
\text { bientôt à sa } \\
\text { place une } \\
\text { jeune fille } \\
\text { tendre, } \\
\text { dévouée, } \\
\text { forte encore, } \\
\text { mais civilisée, } \\
\text { j'aurai gagné } \\
\text { au change. Je } \\
\text { sais déjà que } \\
\text { dans tout } \\
\text { Washington, } \\
\text { je n'aurais } \\
\text { rien pu } \\
\text { trouver qui } \\
\text { valût les } \\
\text { vingt-cinq } \\
\text { dollars que } \\
\text { cette chère } \\
\text { tête ronde } \\
\text { m'a envoyés. }\end{array}$ & $\begin{array}{l}\text { Mon enfant } \\
\text { sauvage me } \\
\text { manquera } \\
\text { peut-être un } \\
\text { peu, mais si } \\
\text { j'ai à la place } \\
\text { une jeune } \\
\text { fille forte } \\
\text { avec un cour } \\
\text { aussi large } \\
\text { que ses } \\
\text { épaules, je } \\
\text { crois que } \\
\text { dans tout } \\
\text { Washington, } \\
\text { je n'aurais } \\
\text { pas pu } \\
\text { trouver } \\
\text { mieux à } \\
\text { acheter avec } \\
\text { les vingt-cinq } \\
\text { dollars } \\
\text { qu'elle m'a } \\
\text { envoyés. }\end{array}$ & $\begin{array}{l}\text { Je regrette un } \\
\text { peu mon } \\
\text { poulain } \\
\text { sauvage; } \\
\text { mais puisque } \\
\text { je trouve à la } \\
\text { place une } \\
\text { femme ser- } \\
\text { viable, coura- } \\
\text { geuse et } \\
\text { tendre, je n'ai } \\
\text { rien perdu } \\
\text { au change. Je } \\
\text { ne sais pas si } \\
\text { c'est la tonte } \\
\text { qui a calmé } \\
\text { notre brebis } \\
\text { galeuse, mais } \\
\text { ce dont je } \\
\text { suis sûr, } \\
\text { c'est que } \\
\text { dans tout } \\
\text { Washington, } \\
\text { je n'ai rien } \\
\text { trouvé d'as- } \\
\text { sez beau à } \\
\text { acheter avec } \\
\text { les vingt-cinq } \\
\text { dollars que } \\
\text { ma brave } \\
\text { petite fille } \\
\text { m'a envoyés. }\end{array}$ & $\begin{array}{l}\text { Si ma petite } \\
\text { sauvageonne } \\
\text { me manque } \\
\text { un peu, je } \\
\text { me réjouis de } \\
\text { trouver une } \\
\text { femme } \\
\text { courageuse, } \\
\text { serviable et } \\
\text { tendre. Je ne } \\
\text { sais si c'est la } \\
\text { tonte qui a } \\
\text { assagi notre } \\
\text { brebis ga- } \\
\text { leuse, mais, } \\
\text { en tout cas, } \\
\text { dans tout } \\
\text { Washington, } \\
\text { je n'ai rien } \\
\text { trouvé d'as- } \\
\text { sez beau à } \\
\text { acheter avec } \\
\text { les vingt-cinq } \\
\text { dollars que } \\
\text { ma brave } \\
\text { enfant m'a } \\
\text { envoyés. }\end{array}$ \\
\hline
\end{tabular}

